\title{
The agonist of JWA gene JAC1 suppresses proliferation of breast cancer through JWA/p38/SMURF1/HER2 signaling
}

\section{Yanlin Ren}

Nanjing Medical University

Dongyin Chen

Nanjing Medical University

Junjie Chen

Nanjing Medical University

Zurong Zhai

Nanjing Medical University

Aiping Li

Nanjing Medical University

\section{Yan Liang}

First Affiliated Hospital of Nanjing Medical University

\section{Yongmei Yin}

First Affiliated Hospital of Nanjing Medical University

\section{Yongqian Shu}

First Affiliated Hospital of Nanjing Medical University

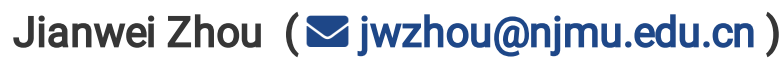

School of Public Health, Nanjing Medical University

\section{Research article}

Keywords: JWA agonist, breast cancer, HER2, E3 ubiquitin ligase, SMURF1

Posted Date: March 5th, 2020

DOI: https://doi.org/10.21203/rs.3.rs-16181/v1

License: (c) (i) This work is licensed under a Creative Commons Attribution 4.0 International License.

Read Full License 


\section{Abstract}

Background The HER2-positive breast cancer accounts for 20-30\% of all breast cancers. The overexpression of HER2 is associated with malignant proliferation and invasiveness in several cancers. Some HER2 targeting drugs are widely used in clinic to block HER2 or its tyrosine kinase and improve the prognosis of patients. However, drug resistance and side effects are still a big challenge worldwide. Our previous studies have demonstrated that JWA down-regulates HER2 expression and inhibits cell proliferation and migration in gastric cancer cells.

Methods The association of JWA with HER2 were analyzed by IHC in 90 tumor sample tissues and paired non-cancerous adjacent normal tissues. The impact of JAC1 on HER2-positive breast cancer cells was studied using colony formation assay. The effect of JAC1 on the localization of HER2 was detected by immunofluorescence microscopy assay. Western blotting, RT-PCR and immunoprecipitation assay were utilized to investigate the mechanisms of JWA regulating HER2. Finally, xenograft mouse models using BT474 cells were established to confirm the effect of JAC1 on tumor growth.

Results In this study, we found that JAC1, a small molecule agonist of JWA gene, dose-dependently suppressed HER2-positive breast cancer proliferation in vitro and in vivo. Importantly, the inhibitory roles of JAC1 was due to the degradation of HER2 overexpression. The mechanistic evidences showed JAC1 increased the ubiquitination of HER2 at the K716 through the E3 ubiquitin ligase SMURF1; further, the activation of SMURF1 was due to reduced expression of NEDD4, an E3 ubiquitin ligase for SMURF1. Finally, we identified that the role of JAC1 on NEDD4 was realized through JWA-p38-GATA-1-NEDD4 axis.

Conclusions These findings suggest that JAC1 may as a novel therapeutic agent to breast cancer through JWA/p38/GATA-1/NEDD4/SMURF1/HER2 signaling axis.

\section{Background}

Despite important advances in cancer research, breast cancer ranks first in morbidity and mortality among female cancers worldwide [1]. The new cases of breast cancer are approximately 2.1 million each year, which remains alarmingly fast. Therefore, breast cancer has become a major health problem and represents a top biomedical research priority [2,3]. Breast cancer is classified into four major molecular subtypes, luminal A, luminal B, HER2-positive breast cancer and normal breast-like cancer [4]. Although several drugs targeting HER2 have improved survival of breast cancer patients, these drugs often develop resistance and eventually resulting in a poor prognosis. Human epidermal growth factor receptor (HER2/ErbB2), a member of the epidermal growth factor receptor (EGFR) family, is negatively or slightly expressed in normal tissues, however it is overexpressed in some breast cancer cases and associated with proliferation and invasiveness of cancer [5]. In the last two decades, the introduction of monoclonal antibodies (e.g. trastuzumab) and tyrosine kinase inhibitors (e.g. Lapatinib) directly against HER2 impressively achieved obvious effects to patients [6-8]. Unfortunately, patients usually developed resistance to anti-HER2 therapies [9-11]. To avoid the side effects of completely blocking HER2, the new 
drug or therapy which could properly down-regulate but not completely block HER2 overexpression in breast cancer cells is greatly expected.

The JWA gene, also known as ARL6IP5, is a multifunctional and cytoskeleton binding protein. The functions of JWA gene are involved in oxidative stress, DNA repair, apoptosis and cell differentiation [12, 13]. Higher JWA expression has also been demonstrated to be a favorable prognostic indicator in several cancers including gastric and breast cancer [14]. The patients with lower expression of JWA in tumor tissues usually receive worse survivals $[15,16]$. JWA negatively regulates HER2 expression and inhibits cell proliferation and migration through transcription and ubiquitination related mechanisms in human GC cells $[17,18]$. However, the role and behind mechanism of JWA on HER2 expression in breast cancer has not yet been determined.

Given that JWA is a broad-spectrum tumor suppressor and negatively regulates HER2 expression in GC cells. To find agonists of JWA gene, we designed a JWA gene promoter sequence based on luciferase reporter gene assay. And we screened the proper candidates from National Compound Library (NCL) which can activate the expression of JWA in HBE cells. In the present study, we used JAC1, a novel agonist of JWA gene, to investigate its effect on HER2 and breast cancer cells both in vitro and in vivo models.

We demonstrated at first time that JAC1 down regulated overexpression of HER2 and suppressed proliferation of breast cancer cells. The mechanistic evidences showed that the function of JAC1 was depend on the presence of the JWA gene in cells; it caused a two-stage ubiquitination modification on target molecules and these actions were driven by p38. Therefore, our data may provide a new concept linked therapeutic potential to HER2 positive breast cancer.

\section{Materials And Methods}

\section{Cell culture}

BT474 and SKBR3 human breast cancer cells were obtained from the American Type Culture Collection (ATCC). The cells were cultured in DMEM supplemented with $20 \%$ and $10 \%$ fetal bovine serum (FBS), respectively. Both cells were maintained with 100 units/ml penicillin and streptomycin (Cellgro, Hemdon, VA) at $37^{\circ} \mathrm{C}$ in a humidified atmosphere containing $5 \% \mathrm{CO}_{2}$.

\section{Western blotting}

Western blot assays were performed on the protocols previously reported [19]. The following antibodies were used: anti- $\beta$-actin; anti-a-tubulin; anti-GAPDH; anti-HA (1;1000, Beyotime, Jiangsu, China); anti-HER2; anti-HER3; anti-p-p38(Thr180/Thr182); anti-Ub (1:1000, CST, USA); anti-JWA (1:100, Laboratory-made); anti-GATA-1; anti-NEDD4; anti-CBL; anti-SMURF1; anti-HER1; anti-HER4; anti-PCNA (1:1000, proteintech, China).

\section{Colony formation assay}


BT474 and SKBR3 cells were seeded into six-well plates with $2 \mathrm{ml} \mathrm{DMEM} \mathrm{containing} \mathrm{20 \%} \mathrm{and} \mathrm{10 \%} \mathrm{FBS,}$ respectively. The cells were treated by 1 or $10 \mu \mathrm{M}$ JAC1 for 10-14 days and not harvested until the visible colonies. Before harvest, BT474 and SKBR3 cells were washed twice with PBS, stained with crystal violet, and only the colonies with $>50$ cells were counted.

\section{Immunofluorescence microscopy assay}

JAC1 or vehicle (DMSO) was added into the BT474 and SKBR3 cell culture dishes for $24 \mathrm{~h}$, followed by washing with PBS 3 times, and then the cells were fixed with methanol for 30 min at room temperature. Next, the fixed cells were washed with PBST (PBS supplemented with $0.5 \%$ Tween-20) and blocking nonspecific signals with 10\% normal goat serum in PBS for $1 \mathrm{~h}$. The cells were incubated with anti-JWA mouse monoclonal antibody (1:200) and anti-HER2 (1:250) overnight at $4{ }^{\circ} \mathrm{C}$. After washing with PBST, the secondary antibodies were incubated with cells for $2 \mathrm{~h}$. The FITC goat anti-mouse IgG (1:100, Beyotime, Jiangsu, China) and CY3 goat anti-rabbit IgG (1:100, Beyotime, Jiangsu, China) was added in and incubated for at least $2 \mathrm{~h}$. After washing three times with PBST, the cells were stained with DAPI (Beyotime, Jiangsu, China) for $20 \mathrm{~min}$. The confocal images of the cells were captured using Zeiss AIM software on a Zeiss LSM 700 confocal microscope system (Carl Zeiss Jena, Oberkochen, Germany).

\section{Quantitative real-time RT-PCR assay}

Total RNA was extracted from harvested cells by using the Trizol reagent (Gibco, USA) according to the manufacturer's instructions. Approximately 500 ng of RNA was used for the reverse transcription reaction with Hiscript Q RT SuperMix for qPCR (Vazyme, Jiangsu, China). The cDNA was amplified with the following primers: 5'-GCCGGTGCTGAGTATGTC-3'(forward) and 5囚-CTTCTGGGTGGCAGTGAT-3》 (reverse)

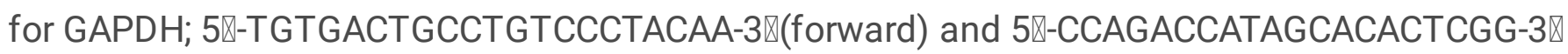

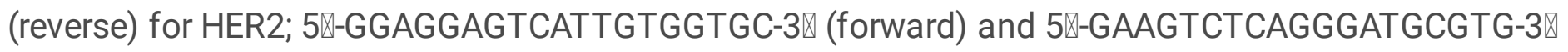
(reverse) for JWA; 5》-TCAGGACAACCTAACAGATGCT-3》 (forward) and 5》-

TTCTGCAAGATGAGTTGGAACAT-3囚 (reverse) for NEDD4. Quantitative RT-PCR was carried out with SYBR Green PCR Master Mix (TaKaRa Bio, Japan) using an ABI Prism 7900 Sequence detection system (Applied Biosystems, Canada).

\section{Plasmids and siRNA transfection}

The construction of Flag-JWA and Ub plasmids were introduced in our previous study [20]. The HA-HER2 plasmid and corresponding mutants were subcloned into a pcDNA3.1 vector (Generay, Shanghai, China) using HindIII/Xhol sites. The siRNA sequences (5'-CGAGCUAUUUCCUUAUCUC-3') used for the JWA and a nonsense control siRNA were synthesized by Ribobio (Guangzhou, China). The plasmids and siRNA were transfected into cells with Lipofectamine 3000 according to manufactures instruction (Invitrogen, Grand Island, NY, USA).

\section{Immunoprecipitation assay}

Before harvest, the cells were washed with PBS twice, and then added pre-cooled immunoprecipitation (IP) buffer and incubated at $4^{\circ} \mathrm{C}$ for $30 \mathrm{~min}$. Next, scraped the cells and collected the cell lysates were 
centrifuged at $12,000 \cdot \mathrm{g}$ for $15 \mathrm{~min}$ at $4^{\circ} \mathrm{C}$. The supernatant was immediately transferred into a new centrifugal tube, and then anti-NEDD 4 antibody, anti-SMURF1 antibody, anti-HER2 antibody and appropriate control IgG (mouse, rabbit IgG, corresponding to the host species of the primary antibody) were added in $500 \mu \mathrm{g}$ total protein and incubated at $4{ }^{\circ} \mathrm{C}$, respectively. After $1 \mathrm{~h}$, cell lysate was mixed with $20 \mu$ l of protein A/G Plus-Agarose (Santa Cruz, USA) at $4{ }^{\circ} \mathrm{C}$ overnight. The complex was washed with pre-cooled PBS 4 times at $1000 \cdot \mathrm{g}$ for $5 \mathrm{~min}$ at $4{ }^{\circ} \mathrm{C}$. Finally, immunoprecipitate was analyzed by SDS-PAGE.

\section{Ubiquitination assay}

BT474 and SKBR3 cells were transiently transfected with Ub for $72 \mathrm{~h}$ and $10 \mu \mathrm{M} \mathrm{JAC} 1$ was added for $24 \mathrm{~h}$, followed by MG132 $(10 \mu \mathrm{M})$ for another $6 \mathrm{~h}$; the cells were harvested for extracting proteins for western blot IP assays. Anti-HER2 antibody (1:200, CST, USA) was added in and cultured at $4{ }^{\circ} \mathrm{C}$ for $1 \mathrm{~h}$, and then Protein A/G Plus-Agarose was added for overnight. Pre-cooled IP buffer was used to wash four times at $1000 \cdot \mathrm{g}$ for $5 \mathrm{~min}$ at $4{ }^{\circ} \mathrm{C}$, and the IP product was examined by western blot.

\section{Tissue microarray and immunohistochemical (IHC) staining}

Human breast cancer tissue microarray (TMA, numbered Brc1802) was purchased from Shanghai Zhuoli Bio, which contains 90 tumor sample tissues and paired non-cancerous adjacent normal tissues. The TMA also provided associated clinical immunohistochemistry and Fish data of HER2. TMAs were immune-stained with anti-JWA monoclonal antibody (1:50). The assessment of the IHC was employed by a semi-quantitative immunoreactivity score (IRS) as reported previously $[12,21]$. Category A documented the intensity of immunostaining as $0-3$ ( 0 , negative; 1 , weak; 2 , moderate; 3 , strong). Category $B$ documented the percentage of immune-reactive cells as $1(0-25 \%), 2$ (26-50\%), $3(51-75 \%)$, and 4 (76$100 \%$ ). Multiplication of category $A$ and $B$ resulted in an IRS ranging from 0 to 12 for each sample, respectively. The concordance for the IRS of the JWA staining scores between the two pathologists was $82(91.1 \%)$ in 90 tumors of the TMA cohort, and the few discrepancies were resolved by consensus using a multi-head microscope. The optimum value of cutoff points of the JWA IRS in this study was 4 . All the samples were classified and analyzed according to these criteria.

\section{Tumor xenografts}

For tumor implantation, $120 \mu$ PBS containing $5 \times 10^{6}$ BT474 cells were injected subcutaneously between the shoulder blades of 6 weeks old female severe combined immune deficiency (SCID)-Balb/c mice from Model Animal Research Center of Nanjing University (Nanjing, China). When tumors were reached an average size of $125 \mathrm{~mm}^{3}$, the mice were randomly divided into 4 groups each with 9 mice, keeping average tumor size similar among groups. Tumor xenografts were measured with calipers every three days, tumor volume $=$ width $^{2} \times$ length $/ 2$. JAC1 $(0,50,100 \mathrm{mg} / \mathrm{kg})$ was administered daily by oral gavage in $0.5 \%$ hydroxypropyl methylcellulose and $0.1 \%$ Tween 80 (Sigma). The animal model was ended when tumors reached average size between 2500 to $3000 \mathrm{~mm}^{3}$ and the animals were then euthanized. Half of each tumor tissue was fixed with $4 \%$ paraformaldehyde and the other half was frozen at $-80^{\circ} \mathrm{C}$ for further 
study. Other organs such as heart, liver, spleen, lung and kidney were also fixed with $4 \%$ paraformaldehyde for routine H\&E staining assay.

\section{Statistical analysis}

Statistical analysis was performed using SPSS software (version 23.0, Inc., Chicago, IL) and GraphPad Prism 6 software (GraphPad Software, Inc., La Jolla, CA, USA). The differences between two independent groups were analyzed by Student's t test (unpaired, two-tailed). For each significantly ectopically expressed molecule, a Kaplan-Meier overall survival and relapse-free survival analysis were performed with a log-rank test. $P<0.05$ was considered statistically significant $\left(* P<0.05\right.$; ${ }^{\star *} P<0.01$; $\left.{ }^{* \star *} P<0.001\right)$.

\section{Results}

\section{JWA negatively regulates HER2 expression and cell proliferation in breast cancer}

To understand the relationship between expressions of JWA and HER2 and breast cancer, firstly, we interrogated the TCGA database. JWA was significantly down regulated in breast tumors compared to that in normal breast epithelium ( $n=1218$; Fig. 1A). The overall survival (OS) was longer in cases with higher JWA expression in tumor tissue than those with lower JWA expression; the OS was shorter in cases with higher HER2 expression than those with lower HER2 expression ( $n=1402$; Fig. 1B-C). To confirm this, we analyzed survival data from GEO database (GSE88770 and GES42568, $n=221$ ). The data also showed that the cases with JWA low and HER2 high expression in tumor tissue had a worst outcome; in contrast, the cases with JWA high and HER2 low expression had a best outcome (log-rank test, ${ }^{*} P<0.05$; $* * P<0.01$; Fig. 1D). We further verified the expressions of JWA and HER2 in protein level in human breast cancer tissue microarray (TMA). As predicted, significant down regulation of JWA was observed in the samples with HER2 overexpression (Fig. 1E). As shown in Additional file 1: Fig. S1A-B, 32/44 cases showed JWA low expression in HER2 positive ones; in contrast, 27/46 HER2 negative cases showed high JWA expression $(n=90 ; P<0.005)$.

To determine if HER2 expression was regulated by JWA, we constructed both Flag-JWA and si-JWA plasmids and their controls and then transfected these plasmids into human breast cancer BT474 cells, respectively. As shown in Fig. 1F, the cells with Flag-JWA showed down-regulation of HER2, however, an up-regulation of HER2 was observed in cells with si-JWA. Therefore, HER2 expression was downregulated by JWA in breast cancer cells. To determine if the down-regulation of HER2 by JWA in breast cancer cells was linked to cell proliferation, we completed colony formation assays. An obvious inhibition of proliferation in BT474 cells with Flag-JWA, however an increased proliferation in BT474 cells with siJWA was determined ( $P<0.005$; Fig. 1G). Confocal imaging assay showed that expression of HER2 on cell membrane was down regulated by Flag-JWA but up-regulated by si-JWA in BT474 cells (Fig. 1H). 


\section{JAC1 down-regulates expression of HER2 and inhibits cell proliferation in breast cancer}

To find the potent agonist of JWA gene in human normal cells, we constructed JWA promoter-containing reporter gene stably transfected HBE cells and completed series high-throughput screening assays from 40,000 compounds in national compound library (Shanghai, China). As shown in Additional file 2: Fig. S2A-B, although all JAC1 JAC6 compounds were indicated responsiveness in screening assays, the colony formation assay showed JAC1 was more effective in inhibiting cell proliferation than the other 5 candidates; Western blot results showed JAC1 was more capable of activating JWA and down-regulating HER2 than other 5 candidates in BT474 cells (Additional file 2: Fig. S2C). The chemical structure formula of JAC1 was shown in Fig. 2A. As shown in Fig. 2B, JAC1 dose-dependently increased JWA however inhibited HER2 expressions at protein levels in both BT474 and SKBR3 cells; the IC 50 of JAC1 on breast cancer cells were then determined (Additional file 3: Fig. S3A-B); the IC ${ }_{50}$ values were $52.28 \mu \mathrm{M}$ in BT474 and $68.46 \mu \mathrm{M}$ in SKBR3 cells, respectively.

To confirm if JWA was necessary to the regulation of JAC1 on HER2 in cancer cells, we constructed JWA knockout (KO) BGC823 human gastric cancer cells by CRISPR/CAS9 technique (the details will describe elsewhere); and an off-target assay of JAC1 on HER2 was completed by JWA KO BGC823 cells. As indicated in Additional file 3: Fig. S3C, JAC1 activated JWA but suppressed HER2 expressions significantly in JWA wild type (WT) BGC823 cells; however, JAC1 had no effects on HER2 expression in JWA KO BGC823 cells. Colony formation assay showed that JAC1 dose-dependently inhibited proliferations in both BT474 and SKBR3 cells (Fig. 2C-D). Confocal imagining showed a dose-dependent reduction of HER2 but induction of JWA expression in both BT474 and SKBR3 cells after treatment with $0,1,10 \mu \mathrm{M}$ of JAC1 for $24 \mathrm{~h}$ (Fig. 2E).

\section{JAC1 down-regulates expression of HER2 via ubiquitin- proteasome pathway}

To determine how JWA down-regulates HER2 in breast cancer cells, we first examined HER2 mRNA levels by RT-PCR after JAC1 treatment. The data showed that JAC1 increased JWA but had non-effect on HER2 at mRNA level in both BT474 and SKBR3 cells (Additional file 4: Fig. S4A). At protein level, when CHX was used for blocking protein synthesis, HER2 degradation was obviously accelerated by JAC1 treatment in both BT474 and SKBR3 cells during the indicating time points (Fig. 3A and Additional file 5: Fig. S5A). We further demonstrated that ubiquitinated HER2 was obviously increased in both BT474 and SKBR3 cells after JAC1 exposure (Fig. 3B-C). To find the potential candidate of E3 ubiquitination ligase to HER2, we searched in web tool of UbiBrowser (ubibrowser.ncpsb.org/) [22]. In this network view the central node was the queried substrate ERBB2 (HER2), and the surrounding nodes were the predicted corresponding E3 ubiquitination ligases. The edge width and surrounding node size were positively correlated with the confidence of prediction. The predicted data shown that NEDD4, CBL and SMURF1 may play important 
roles in ubiquitination degradation of HER2 (Fig. 3D). Among the three candidates, SMURF1 expression was increased but NEDD4 was reduced dose-dependently by JAC1 treatment in both BT474 and SKBR3 cells (Fig. 3E and Additional file 5: Fig. S5B). Similar results were obtained after transfection either with Flag-JWA or si-JWA in SKBR3 cells (Fig. 3F). These data suggest that SMURF1 may be the candidate E3 ubiquitin ligase for HER2. We then completed co-immunoprecipitation experiments to see if SMURF1 can interact with HER2. As we predicted, there was an interaction between HER2 and SMURF1 in BT474 and SKBR3 cells (Fig. 3G and Additional file 5: Fig. S5C). The interactions between HER2 and NEDD4 were also conducted, unfortunately, we obtained a negative result (Additional file 5: Fig.S5D-E).

\section{K716 of HER2 is the ubiquitination target by SMURF1}

To identify the potential target amino acids in HER2 by SMURF1 ubiquitination, we searched http://www.phosphosite.org/; as shown in Fig. 4A, there were 10 candidate ubiquitination sites (lysine, k) in HER2 protein amino acid sequence. We then constructed HER2 mutants for all or separate of these 10 amino acids in HER2 sequence and replaced lysine $(K)$ by arginine $(R)$, respectively. Next, wild type or mutant HER2 expression plasmids were transfected into SKBR3 cells respectively, and followed by JAC1 and $\mathrm{CHX}$ treatment. As a result, only HER2 (K716R) and HER2 (ALL) (all 10 sites mutant) was resistant to CHX-induced reduction of HER2 (Fig. 4B). To confirm this, HER2 (K716R), HER2 (WT) and HER2 (ALL) mutant were transfected into BT474 cells and followed by treatment with JAC1 and CHX. The results obtained in BT474 cells was similar those in SKBR3 cells, JAC1 only accelerated degradation of HER2 (WT) instead of HER2 (K716R) and HER2 (mutant ALL) (Fig. 4C). Then, the ubiquitination of HA-HER2 was attenuated by MG132 treatment in HER2 (K716R) SKBR3 cells compared to those with HER2 (WT) cells (Fig. 4D). Colony formation numbers was increased in HER2 (WT) and HER2 (K716R) BT474 cells compared to control cells $(\mathrm{P}<0.001)$; JAC1 $(10 \mu \mathrm{M})$ treatment obviously suppressed cell proliferation in both HER2 (WT) and control BT474 cells, but no effect on HER2 (K716R) BT474 cells (Fig. 4E-F). Thus, the effect of JAC1 on BT474 cell proliferation was dependent on the presence of amino acid K716 in HER2.

\section{JAC1 modulates SMURF1 through JWA-p38-GATA-1-NEDD4 axis}

Given JAC1 as potent activator of SMURF1, we desired to identify how JAC1 up-regulates SMURF1? As a web tool predicted result, NEDD4 was the most crucial E3 ubiquitin ligase of SMURF1 (Additional file 6: Fig. S6A) since it was dose-dependently down-regulated by JAC1 treatment (Fig. 3E and Additional file 5: Fig. S5B). The expression patterns of SMURF1 and NEDD4 was quite oppositely after JAC1 exposure. In addition, co-IP experiments manifested that SMURF1 exactly interacted with NEDD4 (Fig. 5A-B).

To elucidate how JAC1 down regulates NEDD4 expression, we determined mRNA levels by RT-PCR. The data showed that JAC1 dose-dependently down-regulated mRNA expression of NEDD4 (Fig. 5C-D). Further, the web tool (ALGGEN-PROMO) prediction indicated that several transcription factors may contribute to the JAC1 mediated NEDD4 down regulation (Additional file 6: Fig. S6B). We already knew 
there is non-regulation between ER and HER2. We then focused on both GATA-1 and STAT4 and their relationship to NEDD4 in BT474 and SKBR3 cells. Data showed JAC1 treatment induced a dosedependent increase of JWA, reduction of GATA-1, but no obvious effect on STAT4 (Fig. 5E). Recent studies have revealed that JWA inhibits cell migration by activating MAPK signal pathway [13,23], and TNF-a represses GATA- 1 through activation of p38 MAPK [24]. Here we identified that expression of GATA1 was negatively regulated by JAC1 mediated activation of p38 MAPK (Fig. 5F). To confirm this, p38 inhibitor SB203580 was used to block the role of JAC1 via p38 on NEDD4. Data showed the expressions of p-p38 induced by JAC1 was mostly prevented by SB203580; similarly, JAC1 inhibited GATA-1 expression was mostly reversed under SB203580 treatment in SKBR3 cells (Fig. 5G). To further confirm if this signaling practically mediated the cell proliferation phenotype of JAC1, we completed colony formation assay. The data showed that JAC1 suppressed proliferation of SKBR3 cells was partly reversed by SB203580 (Fig. 5H-I). Taken together, we have revealed the inhibition of JAC1 to proliferation by suppression of HER2 in breast cancer cells.

\section{JAC1 suppresses tumor growth in BT474 breast cancer cell xenograft mice}

To evaluate the translational significance, an in vivo breast cancer BT474 cell xenograft and JAC1 experimental therapy mouse model was conducted. As shown in Fig. 6A, JAC1 dose-dependently inhibited the growth of xenograft tumor. The similar ratio of tumor weight/body weight was shown in Fig. $6 \mathrm{~B}$; the tumor inhibition rate by JAC1 was $31.22 \%$ and $46.21 \%$ in $50 \mathrm{mg} / \mathrm{kg}$ and $100 \mathrm{mg} / \mathrm{kg}$ groups, respectively (Fig. 6C); in addition, compared to the mock group, the solvent showed no significant effect on tumor growth (Fig. 6A-C and Additional file 7: Fig. S7A-B). The picture of isolated xenograft tumor mass of each group was shown in Fig. 6D. To confirm whether the action mechanism of JAC1 on xenograft breast cancer was through the signaling pathways identified in vitro models, the related biomarkers were determined in isolated tumor tissues. As shown in Fig. 6E, expressions of HER2 was dose-dependently reduced by JAC1 treatment; importantly, the mechanistic biomarkers including JWA, pp38, GATA-1, NEDD4 and SMURF1 were indicated corresponding changes in tumor tissues. H\&E staining revealed that JAC1 treatment did not shown obvious injuries on organs including lung, liver, spleen, kidney and myocardium (Additional file 7: Fig. S7C). The serum biochemical parameters indicated a favorable improvement including antioxidant (SOD), liver function (ALT/AST), myocardial cells (CKMB/CK) and lipid metabolisms (TG) after JAC1 treatment (Additional file 8: Supplementary Table 1). Taken together, the in vivo results demonstrated that JAC1 inhibits breast tumor growth in a dosedependent manner; the mechanism of action of JAC1 was consistent between in vivo and in vitro models (Fig. 6F). In conclusion, the agonist of JWA gene JAC1 promoted JWA expression; JWA activated p38 by phosphorylation and suppressed downstream transcription factor GATA-1 and linked E3 ubiquitin ligase NEDD4; the reduced NEDD4 resulted in an overexpression of SMURF1, a specific E3 ubiquitin ligase to HER2 (K716); JAC1 mediated HER2 degradation further suppressed tumor growth in HER2 positive breast cancer. In particularly, JAC1 has a protective effect on major organs and lipid metabolism in mice. 
Since HER2 is a member of the EGFR family, we compared the protein amino acid sequences of four members of the EGFR family (HER1 HER4); and found that K716 is a homologous amino acid and presents in four members (Additional file 9: Fig. S8A). In theory, JAC1 may work for all four members. To confirm this, we determined expressions of HER1, HER3 and HER4 in JAC1 treated SKBR3 cells. As we predicted, JAC1 dose-dependently suppressed expressions of all EGFR members (Additional file 9: Fig. S8B).

\section{Discussion}

The breast cancer patients usually have favorable prognosis and the average five years survival is up to $90 \%$. However, the patients with HER2 overexpression, especially those with cancer metastasis, usually have a poor prognosis [25-27]. The current HER2 targeted therapies are largely improved prognosis of HER2 positive cases, unfortunately, the unavoidable side effects induced by the therapies and resistance are always accompanied to almost all cases and become the biggest challenge in clinic [28]. Although the antibody-drug conjugates (ADC) drugs are indicating more powerful therapeutic effects than HER2target antibody alone [29,30], its side effects are also need to be concerned. The urgent need for HER2 positive breast cancer is a drug or therapy which could degrade the overexpression of HER2 in cancer cells, thereby reversing the excessive proliferation and metastasis in cancer cells induced by HER2. Herein, we identified at first time that JAC1 as a specific agonist of JWA gene, could enhance ubiquitin modification of HER2 at K716 by SMURF1 E3 ligase and accelerate its degradation, thereby inhibiting proliferation of breast cancer cells. The working mode of JAC1 on HER2 was to increase the expression of SMURF1 by reducing the expression of NEDD4. We also determined that the role of JAC1 on NEDD4 was to inhibit the negative transcriptional regulation of GATA-1 through p38 MAPK pathway. JAC1 suppressed the overexpression of HER2 in breast cancer cells to near normal levels and thus maintained the physiological role of HER2 in these cells. JAC1 was also indicated a protective role on liver, myocardial cells, lipid metabolism and anti-oxidant capacity in breast cancer cell xenograft model mice. It is suggested that JAC1 has a potential in drug development and may be as a fundamental therapy to HER2 positive breast cancer.

It is not strange why JAC1 could exert this fundamental role on HER2. We and others have previously reported that JWA gene is not only conserved in evolution but with several essential functions including regulations on cell differentiation, DNA repair, cytoskeleton organization, and metabolisms of sugar and lipid [17, 31, 32]. The biological functions of the JWA gene is achieved largely by regulating of MAPK signaling pathway, which is essential for maintaining basic life activities in organisms [33]. In HER2 overexpressed breast cancer cells, JAC1 could degrade HER2 via p38 MAPK signaling and downstream E3 ubiquitin ligases SMURF1. K716 as a specific target of HER2, was a key point of JAC1 to suppress breast cancer growth.

HER2 is known as a member in EGFR family, which contains four members include EGFR (HER1), HER2 (ErbB2), HER3 and HER4 [34]. HER2 positive breast cancer cells are usually contain homo- or heterodimers of EGFR members such as HER2/HER2 or HER2/HER3 $[35,36]$. In the present study, we also 
identified that K716 is a homologue amino acid in all four EGFR family members (Additional file 9: Fig. S8A); therefore, JAC1 may also be a regulatory agent suitable for all members of EGFR family. In fact, our preliminary data showed that JAC1 could also down-regulate expressions of HER1, HER3 and HER4 in breast cancer cells (Additional file 9: Fig. S8B); the behind mechanisms and translational significances need to be determined.

HER2 is an established therapeutic target in several cancers including breast cancer, several drugs such as trastuzumab, pertuzumab, and lapatinib have been approved for clinical treatment of HER2-positive breast cancer. Trastuzumab works through multiple mechanisms to inhibit tumor growth, including inhibition of downstream signaling by blocking either HER2 homodimerization or ligand-independent HER2/HER3 heterodimerization [37]. Pertuzumab targets a different HER2 epitope than trastuzumab, blocks HER2 dimerization [38]. Lapatinib is an inhibitor to tyrosine kinase of HER2 [8]. However, it is inevitable that some patients will develop resistance to these first-line chemotherapy treatments over time [39]. Recently, the HER2 targeted ADC drug has been approved for clinical use [40]. Unfortunately, all above drugs or therapies are unable to degrade overexpressed HER2 or HER3, and will inevitably produce drug resistance and toxic side effects. Unlike these, in this study, we developed a small molecule compound JAC1 that degrade overexpressed HER2 even all EGFR members. This will provide new insights into EGFR overexpression cancer therapies. Cancer treatments associated with HER2 overexpression or mutations require a new strategy that inhibits HER2 overexpression while leaving normal cells unaffected by the physiological level of HER2, thereby reducing the toxic side effects. EGFR family mutations or rearrangements lead to drug resistance, which is the current challenge for targeted drugs. In theory, JAC1 should also be effective against breast cancer resistant to HER2 rearrangement. In fact, our preliminary data show that JAC1 not only effectively degrades the levels of HER2 in two Herceptin-resistant breast cancer cells, but also inhibits cell proliferation. Further mechanism studies are under way.

Previous reports have demonstrated that NEDD4 is associated with breast cancer progression and predicts a poor prognosis; upregulation of NEDD4 mediates cell migration in lung cancer cells; downregulation of NEDD4 inhibits cell growth and invasion and induces cell apoptosis in bladder cancer cells [41-43]. These evidences support the anticancer function of JAC1 as an indirect inhibitor on NEDD4.

The relationship between p38 and GATA-1 has also reported by Elena Bibikova in hematopoietic progenitors, the activation of the p38 MAPK signaling pathway led to the suppression of GATA-1 [24]. In line with this, JAC1 via p38 MAPK reduced transcription level of GATA-1, this was another key point to the subsequent ubiquitination driven degradation mechanism to HER2. However, the exact mechanism how GATA-1 transcriptionally inhibits NEDD4 expression remains to be investigated.

In conclusion, JAC1, an agonist of JWA gene, was identified as an inhibitor of HER2. The novelty of its action mechanism is the degradation of HER2 overexpression by ubiquitination through p38 MAPK signal pathway. JAC1 may have important translational significance in cancer therapy. 


\section{Conclusion}

The agonist of JWA gene JAC1 inhibits proliferation of breast cancer through JWA/p38/GATA1/NEDD4/SMURF1/HER2 signaling axis.

\section{Abbreviations}

BC: Breast cancer; CHX: Cycloheximide; Co-IP: Co-immunoprecipitation; DMSO: Dimethyl sulfoxide; OS: overall survival; PBS: Phosphate buffered solution; SMURF1: SMAD specific E3 ubiquitin protein ligase 1; TBS: Tris-buffered saline; TMA: Tissue microarrays; Ub: Ubiquitin.

\section{Declarations}

\section{Ethics declarations and consent to participate}

The study was approved by the Ethics Committee of Nanjing Medical University.

\section{Consent for publication}

We have obtained consents to publish this paper from all the participants.

\section{Availability of data and materials}

All other data are available in the main text or the supplementary materials. The datasets used and/or analyzed during the current study are available from the websites mentioned in the text.

\section{Competing interests}

The authors declare that they have no competing interests.

\section{Funding}

This work was supported by the National Natural Science Foundation of China (Nos. 81520108027 , $81673219,81521004,81973156$ and 30930080).

\section{Authors' Contributions}

Conception and design: Y. Ren, J. Zhou

Development of methodology: Y. Ren, J. Chen, Z. Zhai, D. Chen, A. Li, Y. Liang

Acquisition of data: Y. Ren, J. Chen, Z. Zhai, D. Chen, Y. Liang

Analysis and interpretation of data: Y. Ren, J. Chen, Z. Zhai, D. Chen,

Writing, review, and/or revision of the manuscript: Y. Ren, J. Chen, Y. Yin, Y. Shu and J. Zhou 
Administrative, technical, or material support: Y. Ren, J. Chen, Z. Zhai, D. Chen, A. Li

Study supervision: J. Zhou

\section{Acknowledgments}

We thank Ms. Jianping Xiong from Nanjing Medical University for her great support in using confocal microscope.

\section{Disclosures}

None.

\section{References}

1. Bray F, Ferlay J, Soerjomataram I, Siegel RL, Torre LA, Jemal A: Global cancer statistics 2018: GLOBOCAN estimates of incidence and mortality worldwide for 36 cancers in 185 countries. $C A$ : $a$ cancer journal for clinicians 2018, 68(6):394-424.

2. DeSantis C, Howlader N, Cronin KA, Jemal A: Breast cancer incidence rates in U.S. women are no longer declining. Cancer epidemiology, biomarkers \& prevention : a publication of the American Association for Cancer Research, cosponsored by the American Society of Preventive Oncology 2011, 20(5):733-739.

3. DeSantis C, Ma J, Bryan L, Jemal A: Breast cancer statistics, 2013. CA: a cancer journal for clinicians 2014, 64(1):52-62.

4. Cancer Genome Atlas N: Comprehensive molecular portraits of human breast tumours. Nature 2012, 490(7418):61-70.

5. Moasser MM: Targeting the function of the HER2 oncogene in human cancer therapeutics. Oncogene 2007, 26(46):6577-6592.

6. Romond EH, Perez EA, Bryant J, Suman VJ, Geyer CE, Jr., Davidson NE, Tan-Chiu E, Martino S, Paik S, Kaufman PA et al: Trastuzumab plus adjuvant chemotherapy for operable HER2-positive breast cancer. The New England journal of medicine 2005, 353(16):1673-1684.

7. Scheuer W, Friess T, Burtscher H, Bossenmaier B, Endl J, Hasmann M: Strongly enhanced antitumor activity of trastuzumab and pertuzumab combination treatment on HER2-positive human xenograft tumor models. Cancer research 2009, 69(24):9330-9336.

8. Geyer CE, Forster J, Lindquist D, Chan S, Romieu CG, Pienkowski T, Jagiello-Gruszfeld A, Crown J, Chan A, Kaufman B et al: Lapatinib plus capecitabine for HER2-positive advanced breast cancer. The New England journal of medicine 2006, 355(26):2733-2743.

9. Wardley AM, Pivot X, Morales-Vasquez F, Zetina LM, de Fatima Dias Gaui M, Reyes DO, Jassem J, Barton C, Button P, Hersberger V et al: Randomized phase II trial of first-line trastuzumab plus docetaxel and capecitabine compared with trastuzumab plus docetaxel in HER2-positive metastatic 
breast cancer. Journal of clinical oncology : official journal of the American Society of Clinical Oncology 2010, 28(6):976-983.

10. Reid A, Vidal L, Shaw H, de Bono J: Dual inhibition of ErbB1 (EGFR/HER1) and ErbB2 (HER2/neu). European journal of cancer 2007, 43(3):481-489.

11. Giordano SH, Temin S, Kirshner JJ, Chandarlapaty S, Crews JR, Davidson NE, Esteva FJ, GonzalezAngulo AM, Krop I, Levinson J et al: Systemic therapy for patients with advanced human epidermal growth factor receptor 2-positive breast cancer: American Society of Clinical Oncology clinical practice guideline. Journal of clinical oncology : official journal of the American Society of Clinical Oncology 2014, 32(19):2078-2099.

12. Wang S, Wu X, Chen Y, Zhang J, Ding J, Zhou Y, He S, Tan Y, Qiang F, Bai J et al: Prognostic and predictive role of JWA and XRCC1 expressions in gastric cancer. Clinical cancer research : an official journal of the American Association for Cancer Research 2012, 18(10):2987-2996.

13. Bai J, Zhang J, Wu J, Shen L, Zeng J, Ding J, Wu Y, Gong Z, Li A, Xu S et al: JWA regulates melanoma metastasis by integrin alphaVbeta3 signaling. Oncogene 2010, 29(8):1227-1237.

14. Xu L, Cheng L, Yang F, Pei B, Liu X, Zhou J, Zhu Y, Wang S: JWA suppresses the invasion of human breast carcinoma cells by downregulating the expression of CXCR4. Molecular medicine reports 2018, 17(6):8137-8144.

15. Lu J, Tang Y, Farshidpour M, Cheng Y, Zhang G, Jafarnejad SM, Yip A, Martinka M, Dong Z, Zhou J et al: JWA inhibits melanoma angiogenesis by suppressing ILK signaling and is an independent prognostic biomarker for melanoma. Carcinogenesis 2013, 34(12):2778-2788.

16. Wu X, Chen H, Gao Q, Bai J, Wang X, Zhou J, Qiu S, Xu Y, Shi Y, Wang X et al: Downregulation of JWA promotes tumor invasion and predicts poor prognosis in human hepatocellular carcinoma. Molecular carcinogenesis 2014, 53(4):325-336.

17. Qian J, Zhu W, Wang K, Ma L, Xu J, Xu T, Roe OD, Li A, Zhou J, Shu Y: JWA loss promotes cell migration and cytoskeletal rearrangement by affecting HER2 expression and identifies a high-risk subgroup of HER2-positive gastric carcinoma patients. Oncotarget 2016, 7(24):36865-36884.

18. Ma L, Zhu W, Wang Q, Yang F, Qian J, Xu T, Wang S, Zhou J, Shu Y: JWA down-regulates HER2 expression via c-Cbl and induces lapatinib resistance in human gastric cancer cells. Oncotarget 2016, 7(44):71790-71801.

19. Zhou J, Ye J, Zhao X, Li A, Zhou J: JWA is required for arsenic trioxide induced apoptosis in HeLa and MCF-7 cells via reactive oxygen species and mitochondria linked signal pathway. Toxicology and applied pharmacology 2008, 230(1):33-40.

20. Qiu D, Wang Q, Wang Z, Chen J, Yan D, Zhou Y, Li A, Zhang R, Wang S, Zhou J: RNF185 modulates JWA ubiquitination and promotes gastric cancer metastasis. Biochimica et biophysica acta Molecular basis of disease 2018, 1864(5 Pt A):1552-1561.

21. Weichert W, Roske A, Gekeler V, Beckers T, Ebert MP, Pross M, Dietel M, Denkert C, Rocken C: Association of patterns of class I histone deacetylase expression with patient prognosis in gastric cancer: a retrospective analysis. The Lancet Oncology 2008, 9(2):139-148. 
22. Li Y, Xie P, Lu L, Wang J, Diao L, Liu Z, Guo F, He Y, Liu Y, Huang Q et al: An integrated bioinformatics platform for investigating the human E3 ubiquitin ligase-substrate interaction network. Nature communications 2017, 8(1):347.

23. Chen H, Bai J, Ye J, Liu Z, Chen R, Mao W, Li A, Zhou J: JWA as a functional molecule to regulate cancer cells migration via MAPK cascades and F-actin cytoskeleton. Cellular signalling 2007, 19(6):1315-1327.

24. Bibikova E, Youn MY, Danilova N, Ono-Uruga Y, Konto-Ghiorghi Y, Ochoa R, Narla A, Glader B, Lin S, Sakamoto KM: TNF-mediated inflammation represses GATA1 and activates p38 MAP kinase in RPS19-deficient hematopoietic progenitors. Blood 2014, 124(25):3791-3798.

25. Santa-Maria CA, Nye L, Mutonga MB, Jain S, Gradishar WJ: Management of Metastatic HER2Positive Breast Cancer: Where Are We and Where Do We Go From Here? Oncology 2016, 30(2):148155.

26. Chen CY, Yang CY, Chen YC, Shih CW, Lo SS, Lin CH: Decreased expression of stomatin predicts poor prognosis in HER2-positive breast cancer. BMC cancer 2016, 16:697.

27. Slamon DJ, Clark GM, Wong SG, Levin WJ, Ullrich A, McGuire WL: Human breast cancer: correlation of relapse and survival with amplification of the HER-2/neu oncogene. Science 1987, 235(4785):177182.

28. Chun KH, Park JH, Fan S: Predicting and Overcoming Chemotherapeutic Resistance in Breast Cancer. Advances in experimental medicine and biology 2017, 1026:59-104.

29. Thomas A, Teicher BA, Hassan R: Antibody-drug conjugates for cancer therapy. The Lancet Oncology 2016, 17(6):e254-e262.

30. Beck A, Goetsch L, Dumontet C, Corvaia N: Strategies and challenges for the next generation of antibody-drug conjugates. Nature reviews Drug discovery 2017, 16(5):315-337.

31. Chen R, Qiu W, Liu Z, Cao X, Zhu T, Li A, Wei Q, Zhou J: Identification of JWA as a novel functional gene responsive to environmental oxidative stress induced by benzo[a]pyrene and hydrogen peroxide. Free radical biology \& medicine 2007, 42(11):1704-1714.

32. Wang S, Gong Z, Chen R, Liu Y, Li A, Li G, Zhou J: JWA regulates XRCC1 and functions as a novel base excision repair protein in oxidative-stress-induced DNA single-strand breaks. Nucleic acids research 2009, 37(6):1936-1950.

33. Sun Y, Liu WZ, Liu T, Feng X, Yang N, Zhou HF: Signaling pathway of MAPK/ERK in cell proliferation, differentiation, migration, senescence and apoptosis. Journal of receptor and signal transduction research 2015, 35(6):600-604.

34. Yarden Y: The EGFR family and its ligands in human cancer. signalling mechanisms and therapeutic opportunities. European journal of cancer 2001, 37 Suppl 4:S3-8.

35. Hendriks BS, Opresko LK, Wiley HS, Lauffenburger D: Quantitative analysis of HER2-mediated effects on HER2 and epidermal growth factor receptor endocytosis: distribution of homo- and heterodimers depends on relative HER2 levels. The Journal of biological chemistry 2003, 278(26):23343-23351. 
36. Yarden Y, Sliwkowski MX: Untangling the ErbB signalling network. Nature reviews Molecular cell biology 2001, 2(2):127-137.

37. Piccart-Gebhart MJ, Procter M, Leyland-Jones B, Goldhirsch A, Untch M, Smith I, Gianni L, Baselga J, Bell R, Jackisch $\mathrm{C}$ et al: Trastuzumab after adjuvant chemotherapy in HER2-positive breast cancer. The New England journal of medicine 2005, 353(16):1659-1672.

38. Sakai K, Yokote H, Murakami-Murofushi K, Tamura T, Saijo N, Nishio K: Pertuzumab, a novel HER dimerization inhibitor, inhibits the growth of human lung cancer cells mediated by the HER3 signaling pathway. Cancer science 2007, 98(9):1498-1503.

39. Barok $M$, Joensuu $H$, Isola J: Trastuzumab emtansine: mechanisms of action and drug resistance. Breast cancer research : BCR 2014, 16(2):209.

40. Lewis Phillips GD, Li G, Dugger DL, Crocker LM, Parsons KL, Mai E, Blattler WA, Lambert JM, Chari RV, Lutz RJ et al: Targeting HER2-positive breast cancer with trastuzumab-DM1, an antibody-cytotoxic drug conjugate. Cancer research 2008, 68(22):9280-9290.

41. Shao G, Wang R, Sun A, Wei J, Peng K, Dai Q, Yang W, Lin Q: The E3 ubiquitin ligase NEDD4 mediates cell migration signaling of EGFR in lung cancer cells. Molecular cancer 2018, 17(1):24.

42. Wen W, Li J, Wang L, Xing Y, Li X, Ruan H, Xi X, Xiong J, Kuang R: Inhibition of NEDD4 inhibits cell growth and invasion and induces cell apoptosis in bladder cancer cells. Cell cycle 2017, 16(16):15091514.

43. Wan L, Liu T, Hong Z, Pan Y, Sizemore ST, Zhang J, Ma Z: NEDD4 expression is associated with breast cancer progression and is predictive of a poor prognosis. Breast cancer research : BCR 2019, 21(1):148.

\section{Supplementary Information}

Additional file 1: Figure S1. a, Correlation of JWA and HER2 expression in human breast cancer tissue microarray. * $P<0.05 ; * \star P<0.01 ; * \star \star P<0.001$. b, The precise data of (a).

Additional file 2: Figure S2. a, Representative images of the colony formation assay for JAC1-6. b, The number of colonies formed by each group was shown in the histogram. $\mathbf{c}$, The levels of the indicated proteins HER2 and JWA were determined by western blot.

Additional file 3: Figure S3. $\mathbf{a}$ and $\mathbf{b}$, Cell viability of BT474 and SKBR3 cells after JAC1 treatment with indicated doses for $24 \mathrm{~h}$ was determined by CCK8 assay. c, JWA/wild type or JWA/KO cells were treated with JAC1 $(10 \mu \mathrm{M})$ for $24 \mathrm{~h}$. The HER2 and JWA protein levels were determined by western blot.

Additional file 4: Figure S4. a, HER2 and JWA mRNA levels were detected by real-time PCR in BT474 and SKBR3. The values were calculated as $2^{- \text {ddCT }}$, and the relative fold change was compared to the control groups after being normalized to GAPDH. 
Additional file 5: Figure S5. a, SKBR3 cells were treated with the JAC1 $(0,1,10 \mu \mathrm{M})$ for $24 \mathrm{~h}$, followed by $\mathrm{CHX}(100 \mu \mathrm{g} / \mathrm{ml})$ for $0,3,6,9 \mathrm{~h}$. HER2 protein level was determined by western blot. $\mathbf{b}$, The effects of JAC1 on E3 ligase were determined in SKBR3 cells by western blot. c, SKBR3 cells were pretreated with MG132 $(10 \mu \mathrm{M})$ for $6 \mathrm{~h}$, and the endogenous protein-protein interaction between SMURF1 and HER2 were assessed by IP and followed by western blot. $\mathbf{d}$ and $\mathbf{e}$, BT 474 cells and SKBR3 cells were pretreated with MG132 $(10 \mu \mathrm{M})$ for $6 \mathrm{~h}$, and the endogenous protein-protein interaction between NEDD4 and HER2 were assessed by IP with NEDD4 and HER2 antibodies followed by western blot.

Additional file 6: Figure S6. aDNEDD4 is a predicted E3 ubiquitin ligase to SMURF1. b, The predicted transcription factors for NEDD4.

Additional file 7: Figure S7. $\mathbf{a}$ and b, Tumor weight (a), body weight (b), of BT474 xenograft mice model with (1) Mock, (2) JAC1 $0 \mathrm{mg} / \mathrm{kg}$, (3) JAC1 $50 \mathrm{mg} / \mathrm{kg}$ and (4) JAC1 100mg/kg. Compounds were formulated in Error bars represent SEM ( $n=9$ xenograft tumors per treatment group).c, Representative images of H\&E staining sections different organs from mouse model (original magnification, $\times 100$ ).

Additional file 8: Table S1. The serum biochemical indexes (21 parameters) in control and $100 \mathrm{mg} / \mathrm{kg}$ JAC1 treated mice.

Additional file 9: Figure S8. a, Comparison of amino acid sequences of HER1, HER2, HER3 and HER4. * identical amino acid sequence. $\mathbf{b}$, The levels of the HER1,3,4 and JWA proteins were determined by western blot.

\section{Additional file 10ロMicroarray Data.}

\section{Figures}




\section{Figure 1}

A

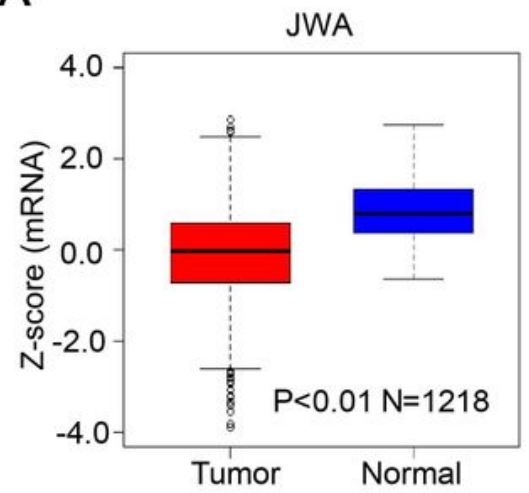

D

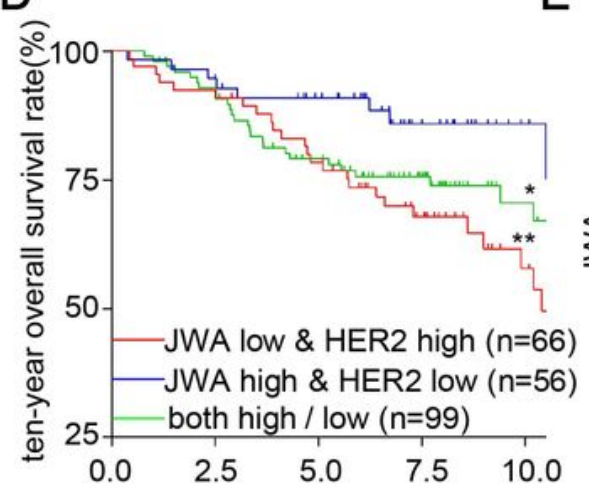

B

E
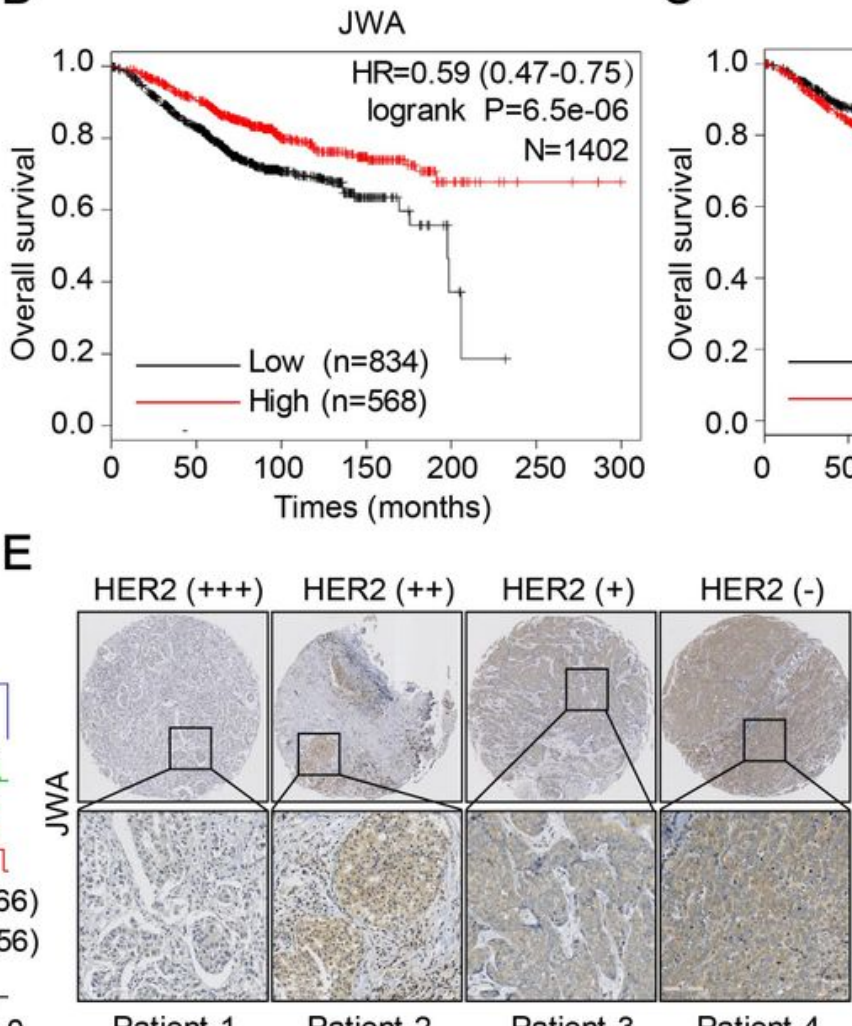

Patient 1

C

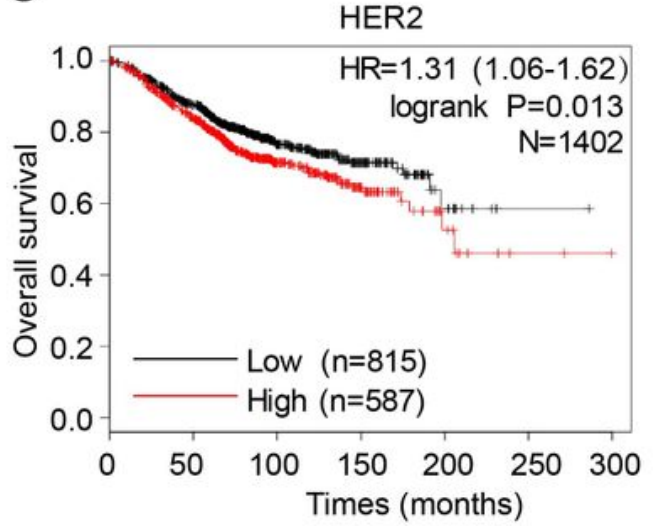

$\mathrm{F}$
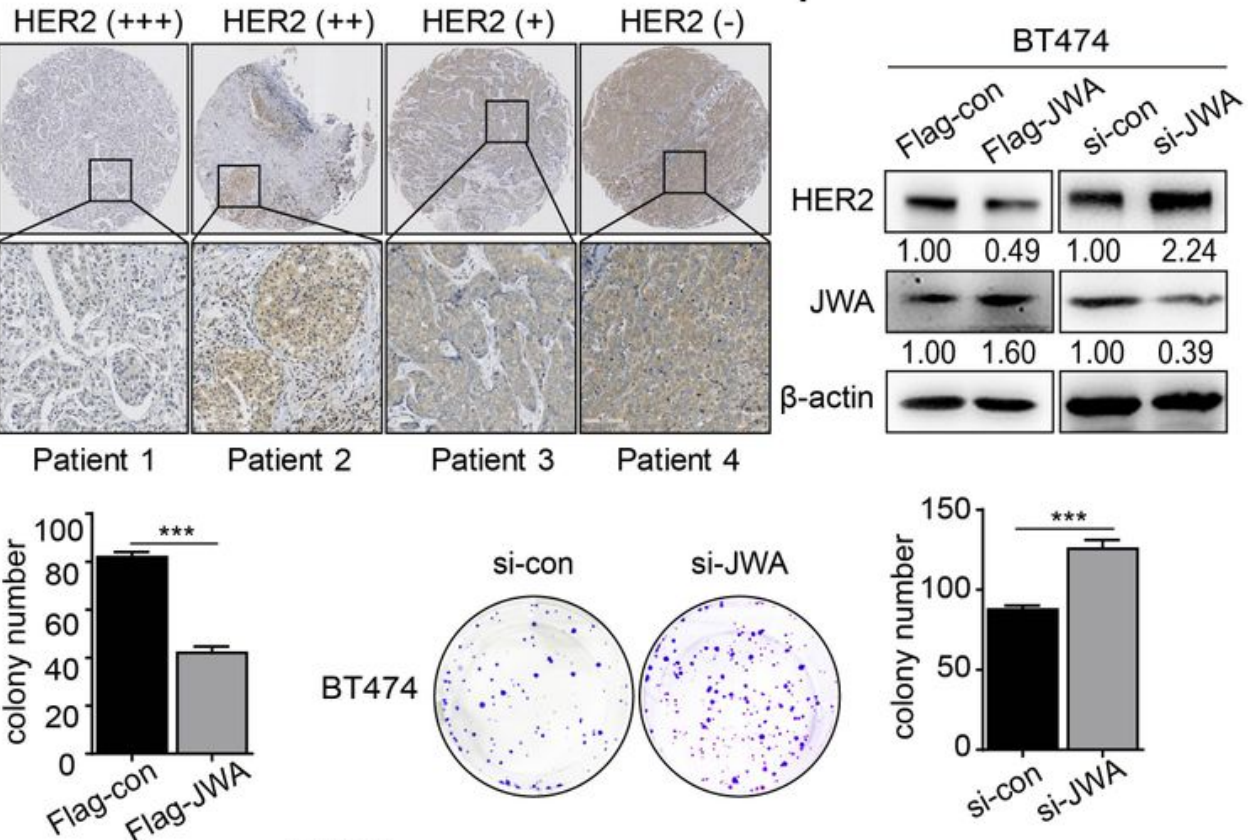

H
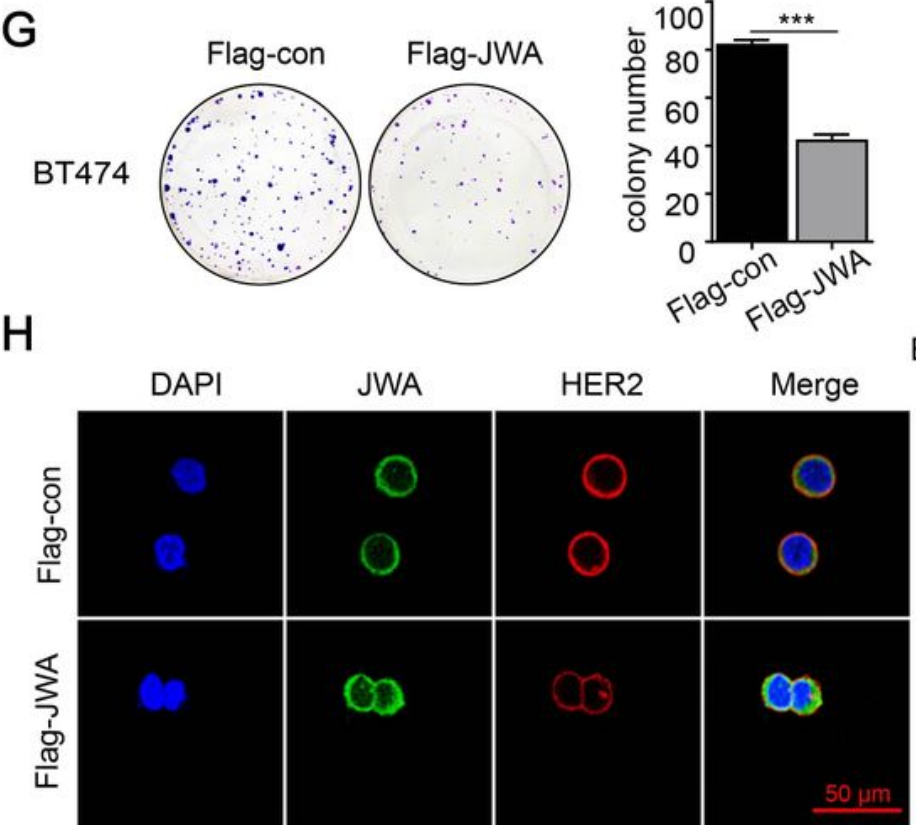

BT474

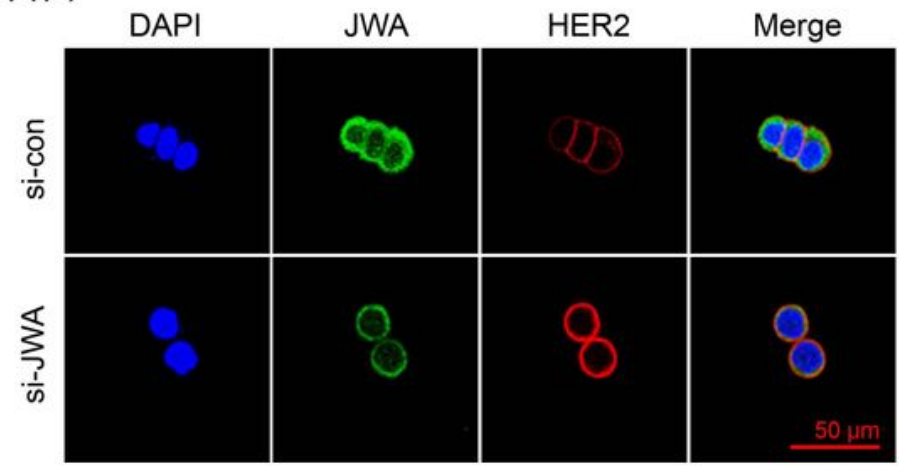

Figure 1

JWA negatively regulates HER2 expression and cell proliferation in BC. a, The mRNA expressions of JWA in $B C$ and adjacent noncancerous tissues in unpaired cohorts (TCGA database, 1099 cancer samples, 119 noncancerous samples). $\mathrm{b}$ and c, Kaplan-Meier curves depicting OS according to the expression patterns of JWA (b) and HER2 (c) in the BC cohort. P values were calculated with the log-rank test. d, Kaplan-Meier OS analysis for BC from GEO datasets (GSE88770 and GES42568). e, Representative 
images of JWA IHC staining in BC lesions with different HER2 scores (original magnification, $\times 2$ and $\times 200$ ). $\mathrm{f}$, Transfection of either Flag-JWA plasmid or si-JWA and their control into BT474 cells for $72 \mathrm{~h}$, the levels of HER2 and JWA proteins were determined by western blot. g, Representative images of the colony formation assay for BT474 after transfection of Flag-JWA or si-JWA and their control cells. h, Immunofluorescence imaging of JWA (green), HER2 (red), nucleus labeled as DAPI (blue), the colocalization of the three signals (merge) in BT474 cells transfected with si-JWA (right) or Flag-JWA for 72 h.

\section{Figure 2}
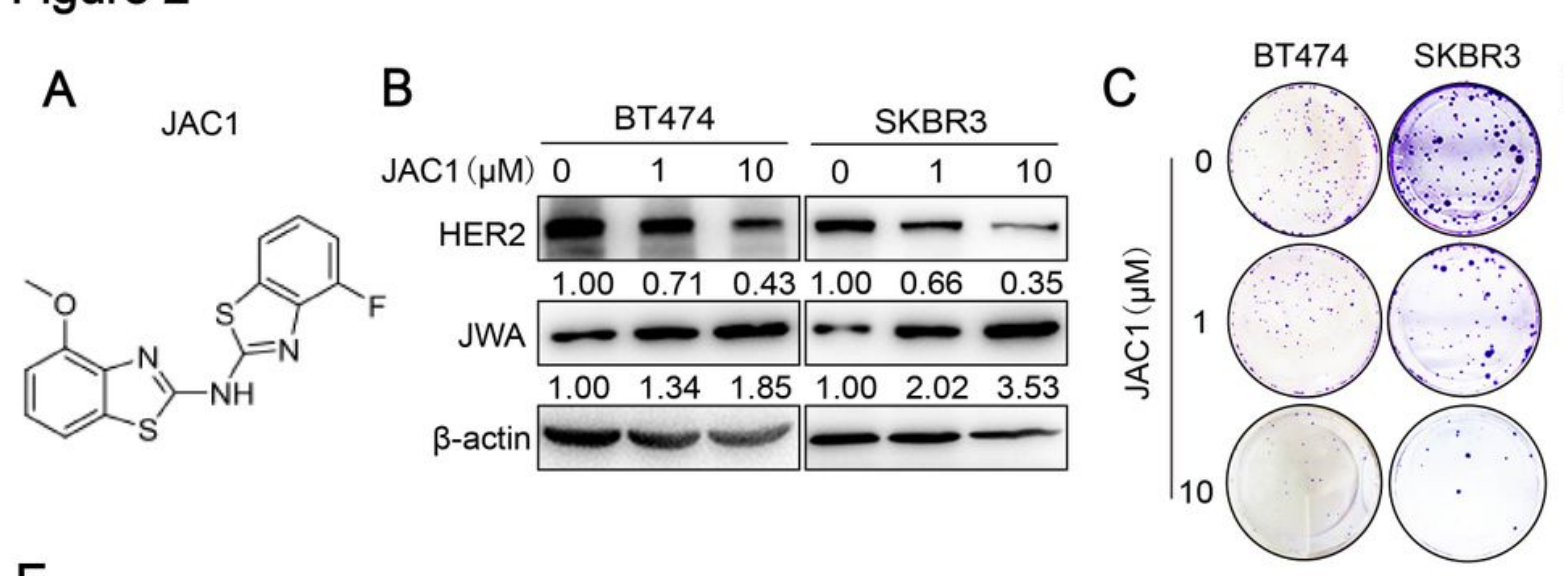

E
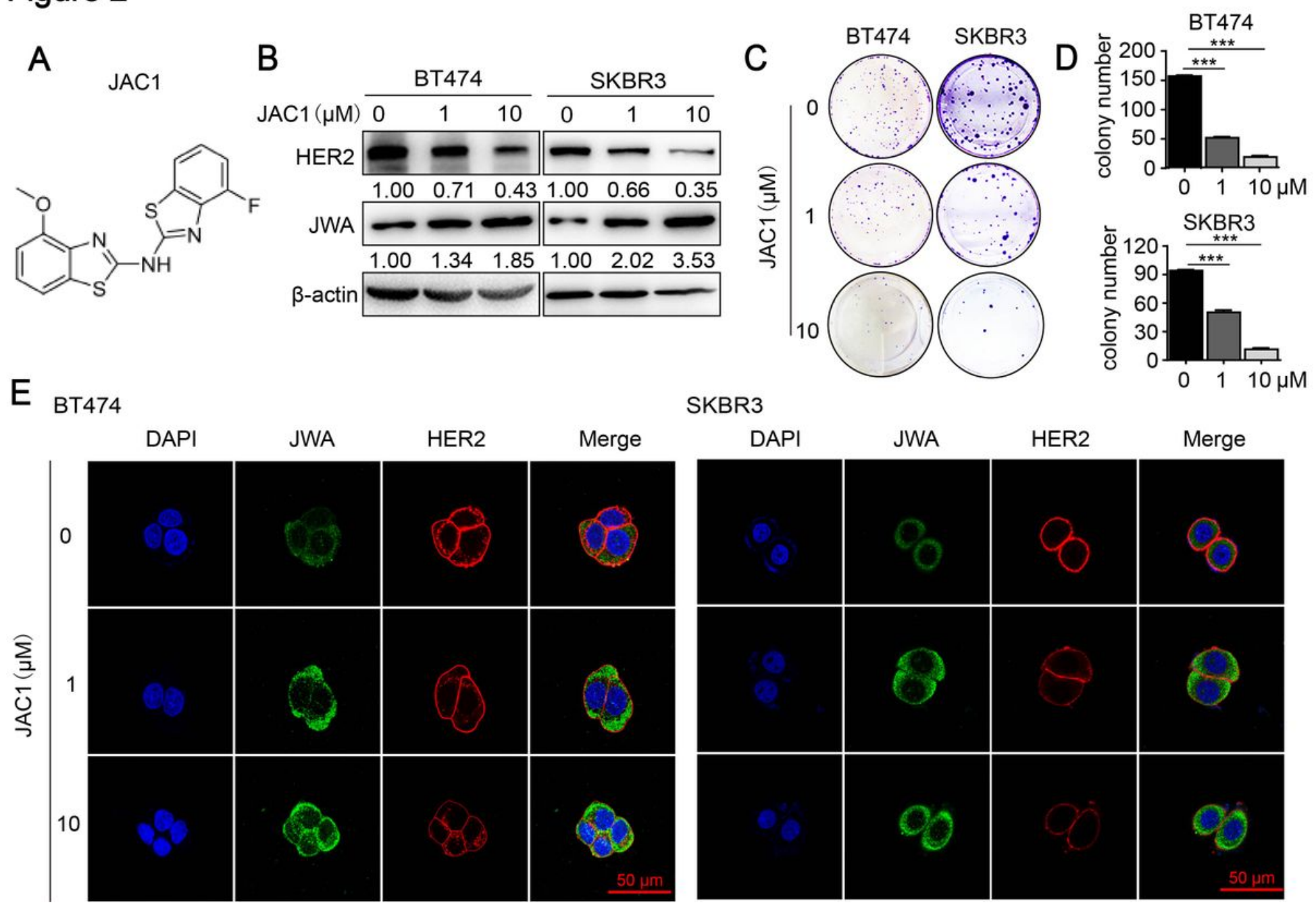

SKBR3

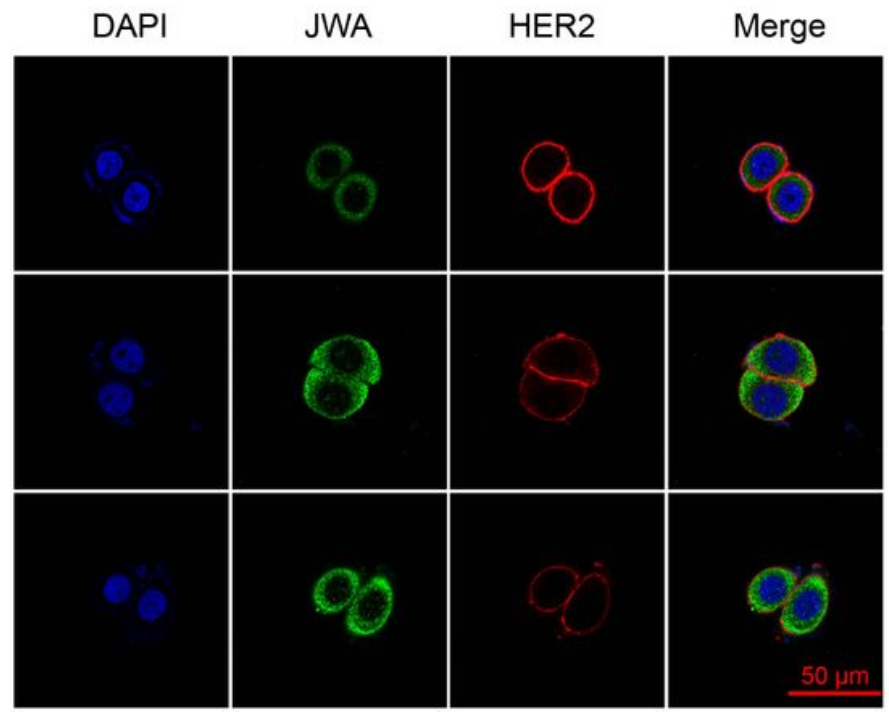

Figure 2

JAC1 down-regulates HER2 expression and inhibits cell proliferation in BC. a, Chemical structure formula of JAC1. b, The levels of the HER2 and JWA proteins were determined by western blot. $c$ and d, Representative images of the colony formation assay for BT474 and SKBR3 cells after treatment with different concentrations of JAC1 $(0,1,10 \mu \mathrm{M})$. e, BT474 and SKBR3 cells were treated with JAC1 $(0,1,10$ $\mu \mathrm{M})$ for $24 \mathrm{~h}$, immunofluorescence imaging showed expressions of JWA (green), HER2 (red), nucleus (blue), the co-localization of the three signals (merge). 


\section{Figure 3}

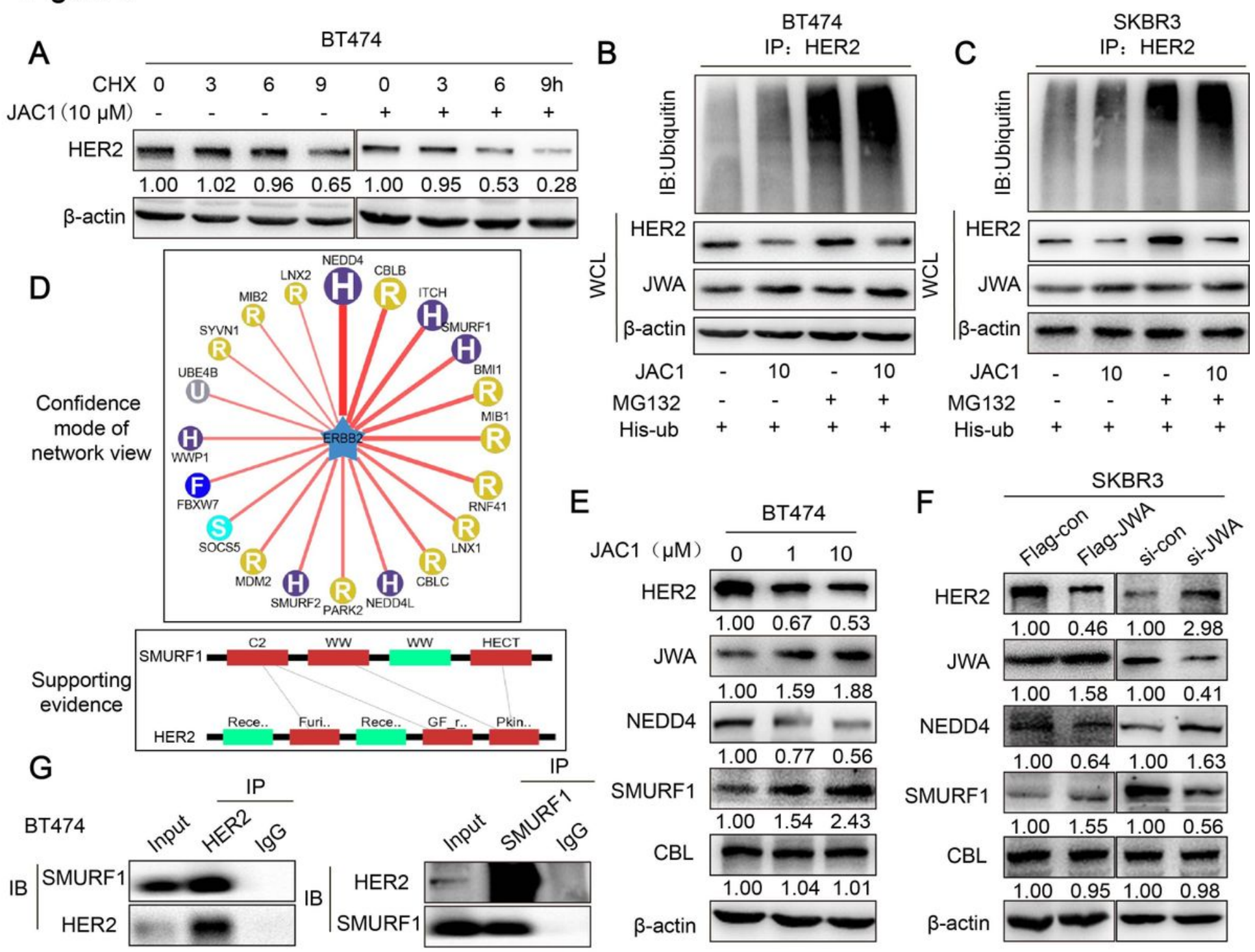

\section{Figure 3}

JAC1 down-regulates HER2 expression via ubiquitin-proteasome pathway. a, BT474 cells were treated with the JAC1 $(0,1,10 \mu \mathrm{M})$ for $24 \mathrm{~h}$, followed by CHX $(100 \mu \mathrm{g} / \mathrm{ml})$ for $0,3,6,9 \mathrm{~h}$. HER2 protein levels were determined by western blot. b and c, Ubiquitination of HER2 was induced by JAC1. Ubiquitination of the HER2 protein was immunoprecipitated and detected a ubiquitin antibody. d, The HER2-targetting E3 ubiquitin ligases were predicted via the public database website (ubibrowser.ncpsb.org/). e, The effects of JAC1 on E3 ligase were determined in BT474 cells by western blot. $f$, The effects of JAC1 on E3 ligase were also confirmed by transfection of Flag-JWA or si-JWA and their control in SKBR3 cells; and the levels of the indicated proteins were determined by western blot. g, BT474 cells were pretreated with MG132 $(10 \mu \mathrm{M})$ for $6 \mathrm{~h}$, and the endogenous protein-protein interaction between SMURF1 and HER2 were assessed by IP and followed by western blot. 


\section{Figure 4}

A

\begin{tabular}{|c|c|c|c|c|c|c|}
\hline K150 & K175 & & & \multicolumn{3}{|c|}{ 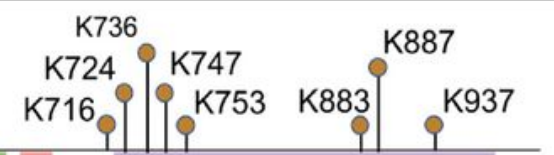 } \\
\hline Recep Ld & Furin-like & Recep Ld & GF recep I & $\mathrm{T}$ & Pkinase Tyr & \\
\hline 100 & 200 & 400 & 600 & 700 & 800 & 1000 \\
\hline
\end{tabular}

B

\begin{tabular}{|c|c|c|c|c|c|c|c|c|c|c|c|c|}
\hline \multicolumn{3}{|c|}{ SKBR3 } & \multicolumn{4}{|c|}{${ }_{150 R}$} & $175 R)$ & hut & $16 R$ & זטה & $\alpha 2^{2(R)}$ & \\
\hline \multicolumn{2}{|c|}{$\mathrm{JAC} 1(10 \mu \mathrm{M})+$} & + & + & + & + & + & + & + & + & + & + & + \\
\hline $\mathrm{CHX}$ & 0 & 9 & 0 & 9 & 0 & 9 & 0 & 9 & 0 & 9 & 0 & $9 \mathrm{~h}$ \\
\hline \multirow[t]{2}{*}{ HER2 } & - & - & - & - & - & - & 一 & - - & - & 一 & - & 一 \\
\hline & 1.00 & 0.36 & 1.00 & 0.50 & 1.00 & 0.76 & 1.00 & 1.36 & 1.00 & 0.72 & 1.00 & 0.38 \\
\hline$\beta$-actin & 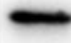 & - & - & - & - & - & - & - & 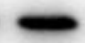 & - & - & - \\
\hline
\end{tabular}

C
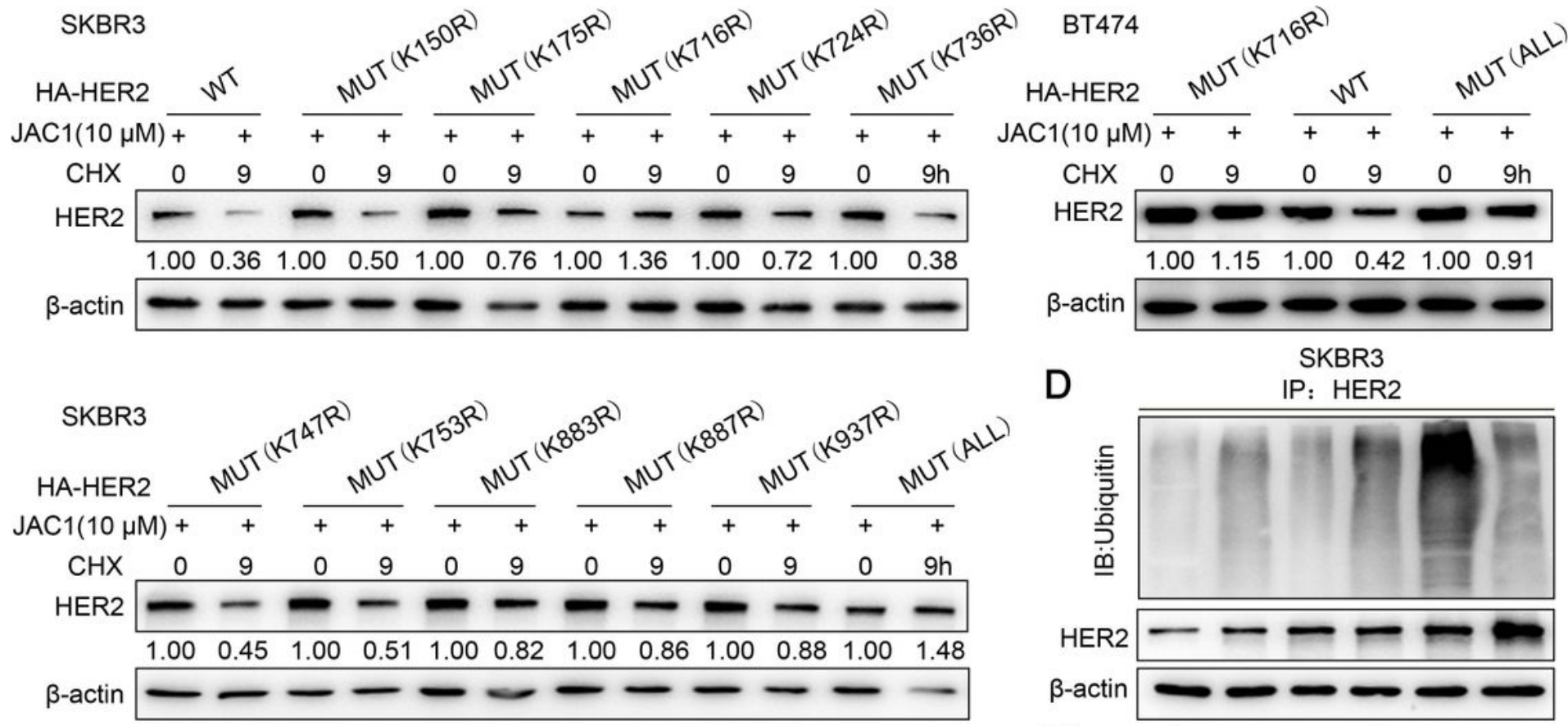

HER2

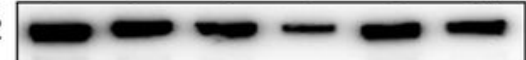

$\beta$-actin $\begin{array}{llllll}1.00 & 1.15 & 1.00 & 0.42 & 1.00 & 0.91\end{array}$

D SKBR3 IP: HER2

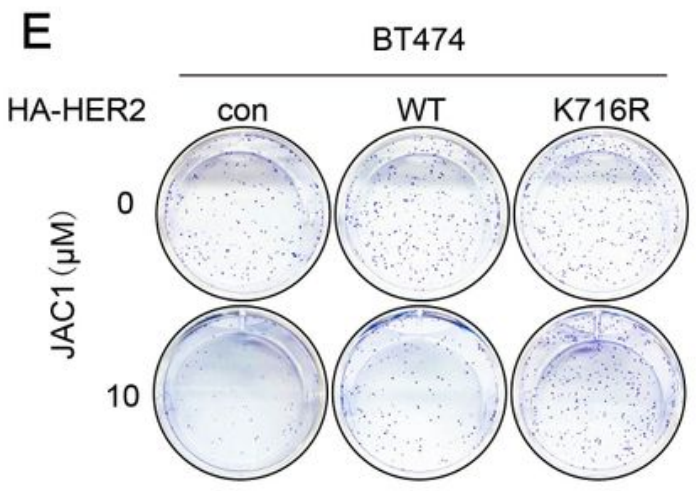

F

HA-HER2
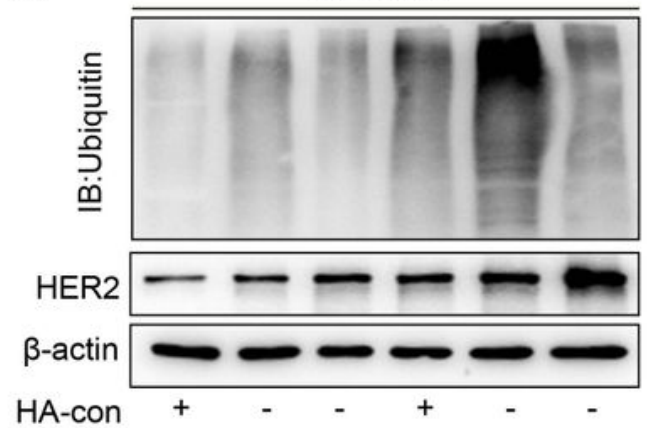

HA-HER (K716R)

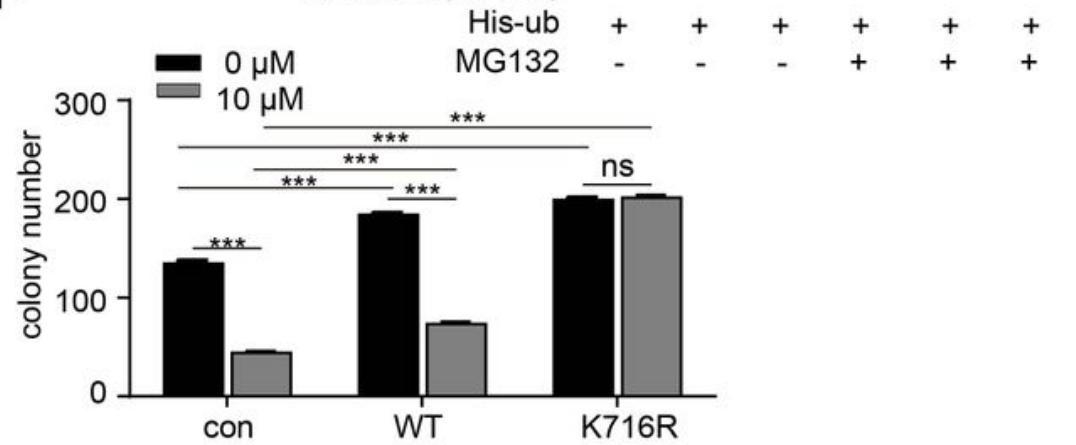

\section{Figure 4}

K716 of HER2 is the ubiquitination target by SMURF1. a, The predicted ten potential ubiquitination targets in HER2. b, SKBR3 cells were transfected with His-HER2 (WT) or 11 mutants (10 separate single amino acid mutants and all 10 mutated amino acids in HER2), treated with the $10 \mu \mathrm{M} \mathrm{JAC} 1$ for $24 \mathrm{~h}$, followed by exposure to $\mathrm{CHX}(100 \mu \mathrm{g} / \mathrm{ml})$ for $9 \mathrm{~h}$. The HER2 expressions were detected by western blot. c, BT474 cells were transfected with HA-HER2 (WT), HA-HER2 (K716) or HA-HER2 (ALL) for $72 \mathrm{~h}$, treated with the $10 \mu \mathrm{M}$ 
JAC1 for $24 \mathrm{~h}$, followed by exposure to $100 \mu \mathrm{g} / \mathrm{ml}$ of $\mathrm{CHX}$ for $9 \mathrm{~h}$; the protein level of HER2 was determined by western blot. d, SKBR3 cells were co-transfected with His-Ub, HA-HER2 (WT) or HA-HER2 (K716) for $72 \mathrm{~h}$, followed by pretreatment with MG132 $(10 \mu \mathrm{M})$ for $6 \mathrm{~h}$. Ubiquitinated HA-HER2 was determined by IP followed immunoblot. e, Colony formation assay was completed for WT/K716R HER2 transfected BT474 cells and followed by JAC1 $(10 \mu \mathrm{M})$ treatment. $\mathrm{f}$, The quantitative data of colony number.

\section{Figure 5}

A

IB

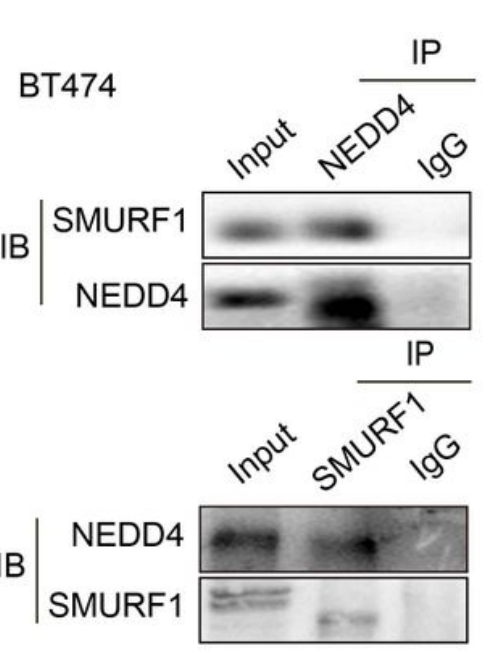

$\mathrm{D}$

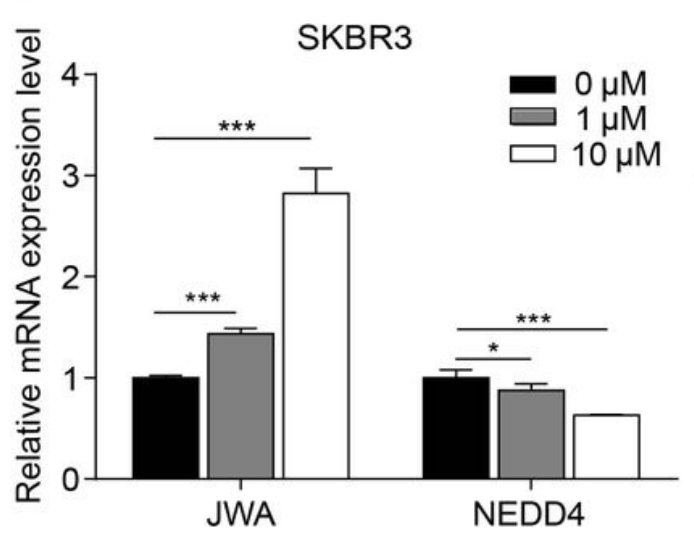

G

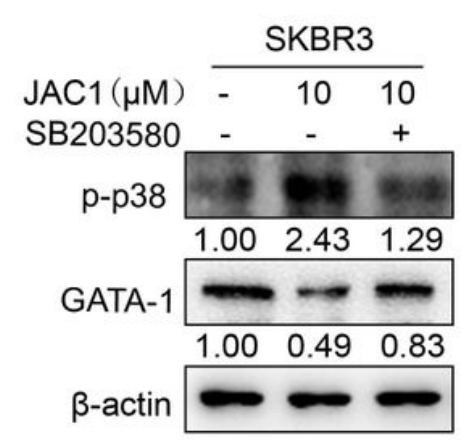

B

IB

IB
SKBR3
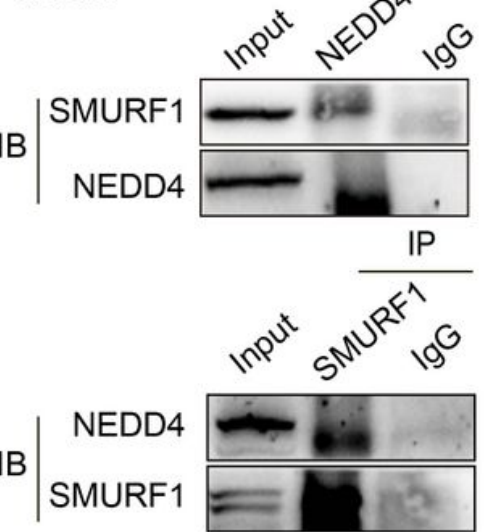

E

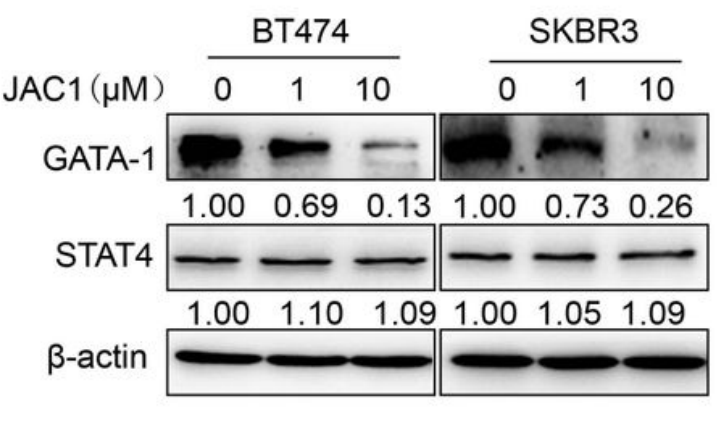

$\mathrm{H}$

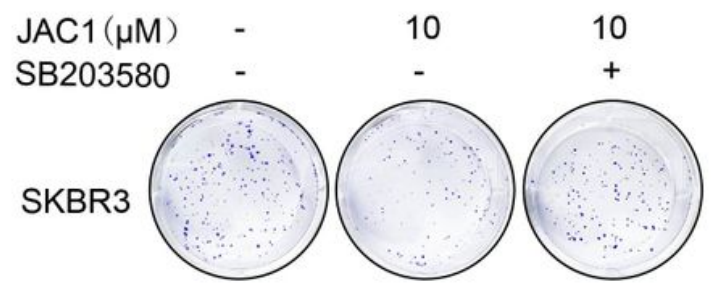

BT474

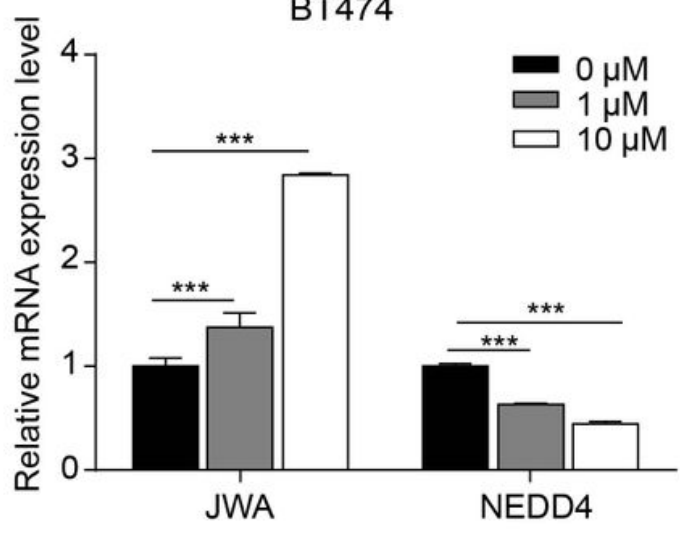

F

JAC1 $(\mu \mathrm{M}) \quad 0 \quad 1 \quad 10$

p-p38

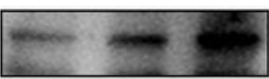

$\beta$-actin $\begin{array}{lll}1.00 & 1.17 & 2.54 \\ - & -\end{array}$

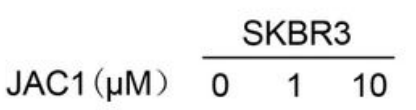

p-p38

$\begin{array}{lll}1.00 & 1.69 & 3.32\end{array}$

$\beta$-actin

I

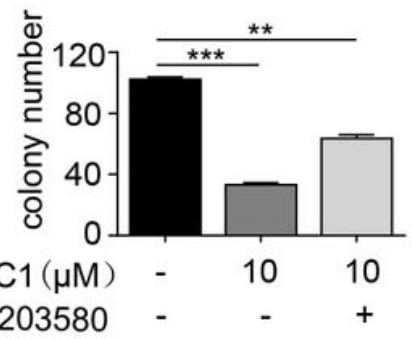

Figure 5 
JAC1 modulates SMURF1 through JWA-p38-GATA-1-NEDD4 axis. a and b, The endogenous proteinprotein interaction between NEDD4 and SMURF1 were identified by IP and western blot in both BT474 and SKBR3 cells. $c$ and d, NEDD4 and JWA mRNA levels were detected by real-time PCR in BT474 and SKBR3. The values were calculated as $\neg 2$-ddCT, and the relative fold change was compared to the control groups after being normalized to GAPDH. e, The regulations of JAC1 on GATA-1 was determined in both BT474 and SKBR3 cells. $f$, JAC1 induced activation of p-p38 was determined by western blot in both BT474 and SKBR3 cells. $g$, The regulations of JAC1 to p38 and GATA-1 was determined by western blot in SKBR3 cells. $h$, The inhibition of JAC1 on colony formation via p-38 MAK pathway was determined in SKBR3 cells. I, The quantitative data of colony number.

\section{Figure 6}

A

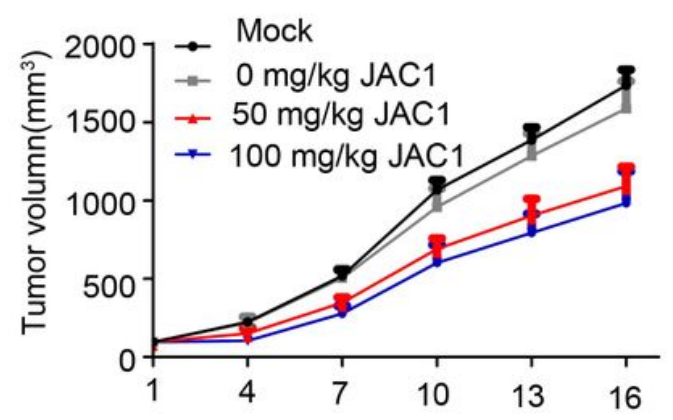

D

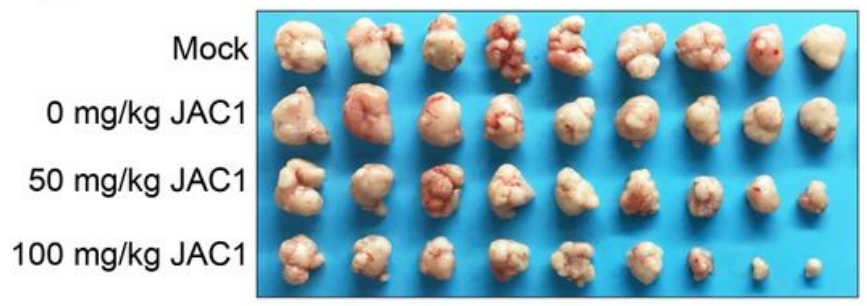

E

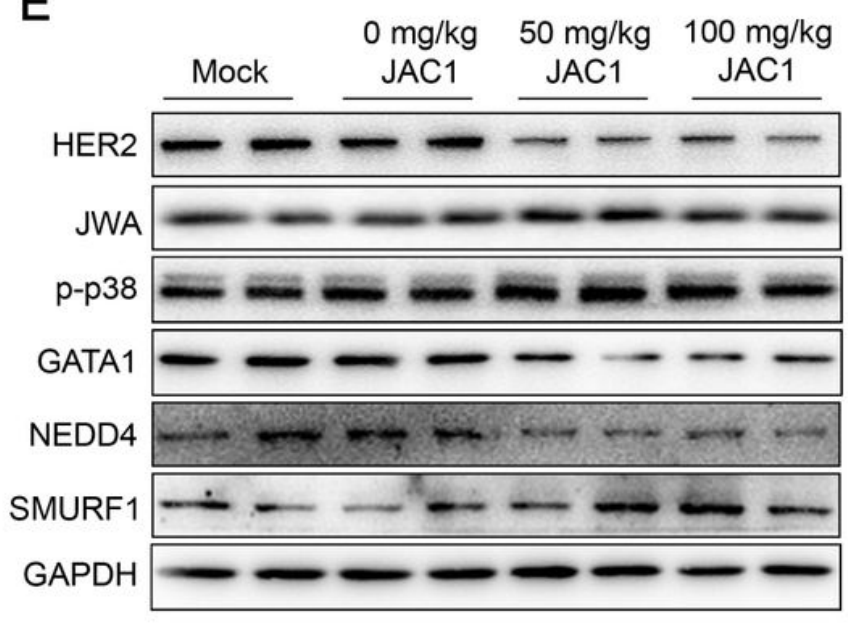

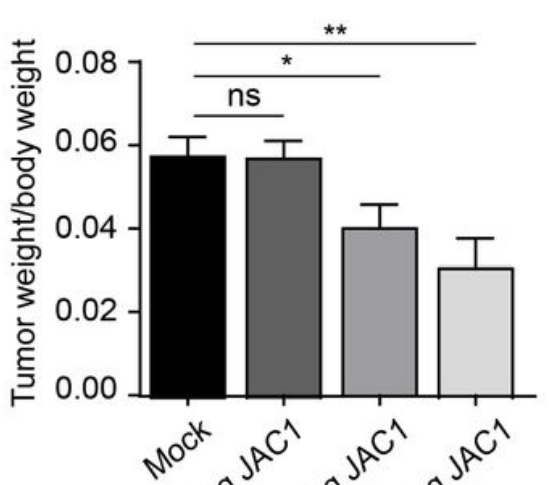

C
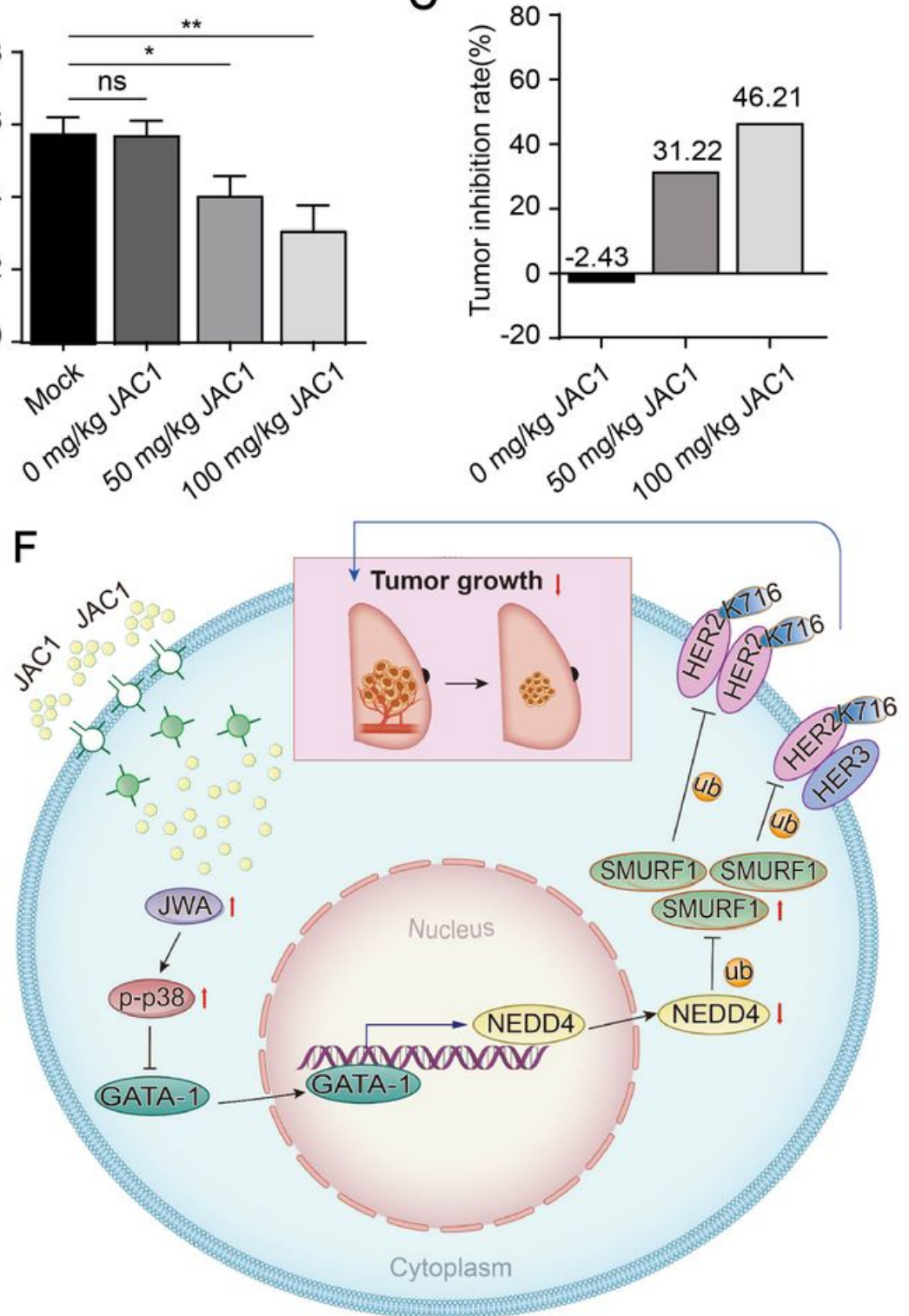

\section{Figure 6}

JAC1 modulates HER2 ubiquitination and inhibits tumor growth in vivo. a, The curve of tumor growth of BT474 xenograft after JAC1 treatment. b-d, The ratio of tumor weight/body weight in each group (b), 
tumor inhibition rate by JAC1 (c), images of the tumor mass in each group (d). e, The expressions of HER2, cell proliferation and mechanistic biomarkers in isolated representative tumor tissues of each group; all the biomarkers were determined by western blot. $f$, A schematic overview how JAC1 degrades HER2 in breast cancer cells. The agonist of JWA gene JAC1 promoted JWA expression; JWA activated p38 by phosphorylation and suppressed downstream transcription factor GATA-1 and linked E3 ubiquitin ligase NEDD4; the reduced NEDD4 resulted in an overexpression of SMURF1, a specific E3 ubiquitin ligase to HER2 (K716); JAC1 mediated HER2 degradation further suppressed tumor growth in HER2 positive breast cancer. ${ }^{*} \mathrm{P}<0.05 ; * \star \mathrm{P}<0.01 ; * \star \star P<0.001$. N.S., no significant differences.

\section{Supplementary Files}

This is a list of supplementary files associated with this preprint. Click to download.

- Microarraydata.xlsx

- Sfig.7.jpg

- Sfig.8.jpg

- Sfig.2.jpg

- Sfig.5.jpg

- Sfig.6.jpg

- Sfig.1.jpg

- Sfig.3.jpg

- Sfig.4.jpg

- SupplementaryTables.docx 